\title{
Review
}

Laura T. Kerr*, Katarina Domijan, Ivor Cullen and Bryan M. Hennelly

\section{Applications of Raman spectroscopy to the urinary bladder for cancer diagnostics}

\author{
Anwendungen der Raman-Spektroskopie für die Krebsdiagnostik der Harnblase
}

\begin{abstract}
Biomolecular changes associated with cancer progression can be identified using Raman spectroscopy, allowing for this technique to be utilized as a non-invasive tool for the diagnosis of bladder cancer. Applications of Raman spectroscopy for diagnostics in real-time have consistently produced higher sensitivities and specificities than current clinical methods. This technique can be applied in vivo during bladder visualization (cystoscopic) procedures as an "optical biopsy" or in vitro to cells obtained from urine cytology specimens. This review follows the evolution of studies in this field from the first in vitro experiment to the most recent in vivo application, identifies how diagnostic algorithms are developed, and provides molecular information associated with the etiology of the biochemical continuum of disease progression. Future prospects for the application of Raman spectroscopy in bladder cancer diagnostics are also discussed.
\end{abstract}

Keywords: Raman microspectroscopy; bladder; cancer diagnostics; Raman fiber-optic probe.

Zusammenfassung: Biomolekulare Änderungen, die mit der Tumorprogression in Verbindung stehen, können mittels Raman-Spektroskopie identifiziert werden, was es erlaubt dieses Verfahren als nicht-invasives Werkzeug zur Diagnose von Blasenkrebs zu verwenden. RamanSpektroskopie-Anwendungen für die Diagnose in Echtzeit haben durchweg höhere Empfindlichkeiten und Spezifitäten als aktuelle klinische Methoden. Das Verfahren kann in vivo während einer Blasenspiegelung (Zystoskopie) als "optische Biopsie" oder in vitro an Zellen, die aus einer Urin-Zytologie gewonnen wurden, eingesetzt werden. Der vorliegende Review-Artikel betrachtet die Entwicklung in diesem Bereich beginnend mit dem ersten In-Vitro-Experiment bis hin zu den neuesten In-Vivo-Anwendungen, zeigt auf, wie Diagnose-Algorithmen entwickelt wurden und liefert molekulare Informationen, die mit der Ätiologie des biochemischen Kontinuums des Krankheitsverlaufs assoziiert sind. Außerdem werden die Zukunftsperspektiven für die Anwendung der Raman-Spektroskopie in der Blasenkrebs-Diagnostik diskutiert.

Schlüsselwörter: Raman-Mikrospektroskopie; Blase; Krebsdiagnostik; faseroptische Raman-Sonde.

\footnotetext{
*Corresponding author: Laura T. Kerr, Department of Electronic Engineering, National University of Ireland Maynooth, Maynooth, Co. Kildare, Ireland, e-mail: lauratheresekerr@gmail.com Katarina Domijan: Department of Mathematics and Statistics, National University of Ireland Maynooth, Maynooth, Co. Kildare, Ireland

Ivor Cullen: University College London Hospitals NHS Foundation Trust, London, UK

Bryan M. Hennelly: Department of Electronic Engineering, National University of Ireland Maynooth, Maynooth, Co. Kildare, Ireland; and Callan Institute, National University of Ireland Maynooth, Maynooth, Co. Kildare, Ireland
}

\section{Introduction}

Bladder cancer is the fourth most common cancer in men, with approximately 200 new cases diagnosed in men and women every day in the USA [1]. As with most solid organ malignancies, early diagnosis, detection and treatment are imperative for good clinical outcomes. Bladder diseases, such as bladder cancer, cystitis and bladder outlet obstruction, are most often diagnosed using invasive procedures. Cystoscopy is the cornerstone of bladder diagnostics for real-time visualization of the bladder mucosa. However, by its nature it is an uncomfortable invasive procedure and is not without significant risk and potential complications. Urine cytology often serves as a useful non-invasive diagnostic adjunct in the diagnosis of bladder cancer. Examination of voided urine or bladder washing specimens for exfoliated cancer cells has high sensitivity in high-grade tumors but low sensitivity 
in low-grade tumors [2]. It has been shown that delays in the process of diagnosis leads to unsatisfactory outcomes and adverse effects for the patient involved [3, 4]. Therefore, there is a real clinical need to develop and integrate a non-invasive, sensitive and specific technique to assist in bladder cancer diagnosis and differentiation between low- and high-grade diseases.

Optical diagnostic tools in medicine are becoming more popular in recent times because they can provide fast, highly accurate results without damaging the biological samples with toxic chemical markers. Raman spectroscopy is one of the most promising optical diagnostic tools that can be applied to the urinary bladder as it is able to detect changes in the molecular composition of tissue samples in real time, which is not only useful for discriminating between different grades and stages of disease, but which may also provide epigenetic clues as to the etiology of the malignancy. This information is obtained by measuring changes in the wavelength of light as it is scattered from the tissue (or cell) sample. Raman scattering occurs when an incident laser photon interacts with a molecular bond in the sample, and the emitted photon has a different energy than the incident one, which results in a shift in the wavelength of the photon. This shift in wavelength provides information about the type of molecular bond present in the sample being interrogated. The Raman spectra obtained from tissue or cells can be inputted to trained statistical multivariate algorithms in order to diagnose bladder cancer with high sensitivity and specificity. The application of Raman spectroscopy for the detection of bladder disease is a highly accurate diagnostic tool that can be applied in vivo, in vitro and ex vivo using a variety of spectroscopic systems, which will be further discussed later.

This paper reviews the contributions over the past decade on the application of Raman spectroscopic techniques for improving the accuracy of detecting bladder cancer and some other forms of bladder disease. The existence of review papers previously published in the application of Raman spectroscopy to urology [5] and to cancer diagnostics in general [6] are acknowledged. Here the focus is only on bladder disease which allows us to provide a significantly deeper level of detail on this subject matter. This paper is intended to be legible for both the biomedical physicist and the clinician.

The results achieved for the diagnosis of bladder cancer using Raman spectroscopy to date are significantly better than current clinical methods, particularly for urine cytology. Presently, white light cystoscopy, which is most often used to diagnose bladder cancer, has sensitivities and specificities between $62-84 \%$ and $43-98 \%$, respectively [7], whereas Raman-based cystoscopy has shown a sensitivity of $85 \%$ and specificity of $79 \%$ within the first in vivo study [8], and sensitivity and specificity of $85.7 \%$ and $100 \%$ using a confocal fiber-optic probe ex vivo [9]. Fluorescence cystoscopy using 5 -aminolevulinic acid (5-ALA) or its hexyl ester, hexaminolevulinate (HAL), has previously been shown to achieve sensitivities and specificities of $76-97 \%$ and $43-79 \%$ respectively [10]; when fluorescence and Raman spectroscopy modalities are combined, $100 \%$ sensitivity and specificity has been demonstrated [11]. Urine cytology has the advantage of being entirely non-invasive but its low sensitivity, particularly for low-grade tumors, which represent the bulk of cases, has meant that it is generally not used as a primary diagnostic tool. For high-grade tumors, sensitivities of $52-78 \%$ are obtained using urine cytology, but for lowgrade tumors, this sensitivity decreases significantly to 0-50\%. However, in the first patient study Raman cytology has been shown to successfully diagnose between normal, low-grade and high-grade bladder cells with a sensitivity of $92 \%$ and specificity of $91 \%$ [12]. Interesting new approaches such as modulated Raman spectroscopy have been successfully applied to bladder cancer cell lines [13] and may hold the potential to further improve upon the diagnostic sensitivity and specificity of Raman cytology.

The breakdown of this paper is as follows:

- Firstly, an introduction to bladder pathology will be given in Section 2 with information on how bladder cancer is currently diagnosed and classified by pathologists based on histological grading or pathological staging systems, and also on how nonmalignant bladder diseases are identified.

- This is followed by an introduction to Raman spectroscopy, the physics involved in this scattering process, and an explanation into why this technique can be utilized as a means of diagnosing bladder cancer in Section 3. There are three main spectroscopic systems associated with the application of Raman spectroscopy for diagnostics: conventional Raman microspectroscopy, Raman spectroscopy with a fiber-optic probe and Raman optical tweezers. A detailed description of each of these methods will be discussed in Sections 3.2, 3.3 and 3.4, respectively, with an emphasis on how to integrate these systems into a standard microscope at a minimal cost.

- Section 4 reviews the various contributions on the application of Raman spectroscopy to bladdercancer diagnostics over the last decade. This starts by discussing the diagnostic classification algorithms and how the performance of these models is 
determined using cross validation methods. Section 4.2 summarizes all previous applications of Raman spectroscopy to diagnose bladder biopsy and tissue samples in vitro and ex vivo. Section 4.3 then focuses on the use of Raman fiber-optic probes integrated into cystoscopes for real-time cancer diagnostics in vivo. Both of these techniques have been shown as being highly accurate for discriminating between healthy and cancerous bladder tissue, as well as identifying the exact stage/grade of disease present in a minimally invasive manner. Section 4.4 proceeds to describe the completely non-invasive technique of combining Raman spectroscopy with urine cytology. Here, Raman spectroscopy has been successfully applied to cells obtained from voided urine for diagnosis of bladder carcinoma with high sensitivities and specificities.

- Finally, Section 5 demonstrates the use of Raman spectroscopy as an aid for investigating the etiology of bladder cancer. Raman spectroscopy has the ability to identify the biochemistry of a tissue sample, and has been utilized to identify the molecular changes associated with the biochemical continuum of disease as it progresses from healthy to a high-grade bladder carcinoma.

\section{Bladder disease and diagnosis}

\subsection{Diagnosis of bladder pathology}

The main bladder pathologies associated with this review are cystitis (i.e. inflammation of the bladder), bladder outlet obstruction, benign bladder tumors, dysplasia/ atypia (i.e. abnormal cells) and bladder cancer, which are generally diagnosed using cystoscopy, aided by urine cytology. Cystoscopy is an invasive endoscopic procedure of the urinary bladder carried out via the urethra, however it is sometimes indeterminate and there are variables that can result in false negative results.

Bladder mucosa inflammation (cystitis) is commonly encountered in individuals with a urinary tract infection and individuals with indwelling catheters, and carcinoma in situ (CIS), a particularly aggressive form of non-invasive bladder cancer with a high propensity to progress to muscle invasive disease, also appears as inflamed bladder lining. Cytology is useful when a high-grade carcinoma (HGC) or CIS is present but its use in the diagnosis of low-grade carcinoma (LGC) is very limited owing to its low level of sensitivity [14]. As a standard procedure, cystoscopy is performed using white light. However, the use of white light can lead to missing lesions that are present but not visible, which is why new technologies are being developed.

Photodynamic diagnosis (fluorescence cystoscopy) is performed using violet light after intravesical (intrabladder) instillation of 5-ALA or HAL. It has been confirmed that fluorescence-guided biopsy and resection are more sensitive than conventional procedures for the detection of malignant tumors, particularly for CIS of the bladder [15]. This paper reviews the advantages of using Raman spectroscopic techniques for the diagnosis of bladder pathologies in real-time with high sensitivities and specificities.

\subsection{Classification of bladder carcinoma}

Approximately $90 \%$ of all bladder cancers are diagnosed as urothelial carcinoma (UC), otherwise known as transitional cell carcinoma (TCC) [16]. UC develops in the urothelium of the bladder wall, and is the second most common malignancy of the genitourinary tract [17]. The remaining $10 \%$ of bladder cancers consist of squamous cell carcinoma (SCC), adenocarcinoma and small cell carcinoma. Almost $80 \%$ of patients with TCC present with non-muscle-invasive or superficial bladder cancer, the remainder being infiltrative, invading the detrusor muscle and potentially spreading to loco-regional lymph nodes and viscera [18]. There are two main diagnostic classification systems for UCs based on histological grading and pathological staging. Histological grading represents the degree of differentiation present between the carcinoma cells and normal urothelial cells which can be used to predict the rate at which the carcinoma cells are multiplying. Pathological staging is used to assess the extent of cancer invasion into the bladder wall in order to determine if the disease has spread.

\subsubsection{Histological grading}

The histological grade of a UC tumor is determined under a microscope using a specific set of criteria to establish how much the tumor resembles normal (healthy) tissue. Within histological grading, there are two systems in use; the World Health Organization (WHO) 1973 grading system and the WHO 2004 grading system. Most histopathologists still use the WHO 1973 system, which is based on a three-tiered grading system as summarized in Table 1. Grade 1 represents well differentiated cells that are slow 
growing and look similar to healthy bladder cells, Grade 2 cells are moderately differentiated, and Grade 3 are poorly differentiated, fast growing cells that look very different to healthy cells [19], as illustrated in Figure 1.

Histological grading is often criticized given the subjective nature of this test as it is based on the pathologist's perception of cell differentiation. Generally, diagnosis is made by two or more pathologists per sample in order to avoid misclassification, however there is still often inter-observer variation (between pathologists) and intraobserver variation (from one pathologist at different times) [20]. Thus, this method of classification is time-consuming with a high degree of variability. The future trend is leaning towards the use of the newer system (WHO 2004), which is based on simply grading cancers as low or high grade, thus reducing inter-observer variation.

\subsubsection{Pathological staging}

Pathological staging is based on the tumor-node-metastases (TNM) system, whereby the pathologist assesses the location of the tumor (T), if the cancer has spread to nearby lymph nodes $(\mathrm{N})$ and the extent of metastases (M). A number is assigned to each value ranging from 1 to 4 , where higher numbers indicate a more aggressive, invasive carcinoma [21]. The tumor (T) value, which is the most important value in this review, represents the location of the tumor with respect to the inner lining of the bladder

Table 1 Description of bladder cancer cells corresponding to each histological grade.

\begin{tabular}{ll}
\hline $\begin{array}{l}\text { Histological } \\
\text { grade }\end{array}$ & Description of cancer cells \\
\hline GX & Unable to determine the extent of differentiation \\
G1/low grade & Well differentiated \\
G2 & Moderately differentiated \\
G3/high grade & Poorly differentiated \\
\hline
\end{tabular}

A

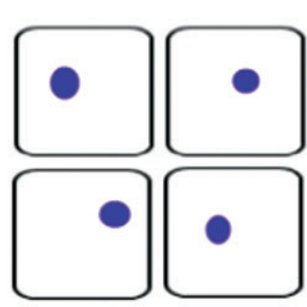

B

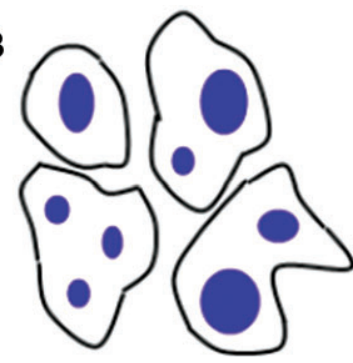

Figure 1 Basic representation of the differences between normal bladder cells (A) and poorly differentiated cancer cells (B). wall. Stages Ta, TIS (referring to carcinoma in situ) and T1 are non-muscle-invasive UCs, with Ta and TIS both confined to the urothelium. Most Ta and T1 tumors are low grade and are less likely to become muscle-invasive. TIS is a flat, high-grade, non-invasive UC. Macroscopically, CIS can be missed at cystoscopy or be considered as an inflammatory lesion if not biopsied. A summary of each pathological stage can be seen in Table 2, and Figure 2 illustrates the extent of the growth of the tumor with respect to the inner bladder wall lining.

\subsection{Non-malignant bladder pathology}

Other non-malignant pathologies that may be diagnosed in the urinary bladder using Raman spectroscopy include cystitis, damage to the bladder wall caused by bladder outlet obstruction, dysplasia/atypia and benign tumors.

Cystitis is essentially inflammation of the bladder, usually as a result of an infection of the urinary system but it is often difficult to detect using conventional methods. Bladder outlet obstruction, most commonly caused from an enlarged prostate or urethral structure, may result in progressive deterioration of the bladder and ultimately kidney function, and it is often difficult to determine the extent of the loss of functionality that will be present after surgical removal of the obstruction. Raman spectroscopy has the potential to improve the diagnosis of bladder functionality due to urethral obstruction by analysing the bladder wall composition. Raman spectroscopy can also be applied to regions of dysplasia/atypia and benign tissues, and be able to clearly distinguish between these and cancerous tissue cells.

\section{Raman spectroscopy}

\subsection{Introduction to Raman spectroscopy}

Raman spectroscopy is a powerful tool for the analysis of the biomolecular composition of biological samples. This technique is based on the inelastic scattering of monochromatic (laser) light; this means that when light interacts with the biological sample, the emitted photons have a different energy to the incident photons. Only about one in $10^{7}$ photons will undergo Raman scattering [22], the rest will undergo Rayleigh scattering as normal. Figure 3 compares the energy differences between photons emitted during Rayleigh scattering and Raman scattering, whereby there is no change in energy between incident 
Table 2 Description of bladder cancer tumor corresponding to each pathological stage [21].

\begin{tabular}{ll}
\hline Pathological stage & Description of tumor \\
\hline T0 & No primary tumor \\
Ta & Non-invasive papillary tumor, confined to urothelium \\
TIS/CIS & High-grade, flat tumor, confined to urothelium \\
T1 & Invasive tumor; invades lamina propria (connective tissue region) \\
T2 & Muscle invasive tumor \\
& T2a - tumor invades inner half of muscle layer \\
& T2b - tumor invades outer half of muscle layer \\
T3 & Tumor invades perivesical fat layer beyond the muscle region \\
& T3a - microscopically \\
& T3b - macroscopically \\
T4 & Tumor invades nearby tissues such as the prostate, vagina, uterus, pelvic wall or abdominal wall \\
& T4a - tumor invades prostate, uterus or vagina \\
& T4b - tumor invades pelvic wall or abdominal wall \\
\hline
\end{tabular}

TIS/CIS, carcinoma in situ.

photons and Rayleigh scattered photons, but Raman scattered photons either gain energy (Anti-Stokes) or lose energy (Stokes) during this scattering process. The magnitude of the change in energy seen in the Raman photons is dependent on the rotational and vibrational energies of the molecules in the biological sample [23]. By analyzing these Raman photons, it is possible to determine the molecular composition of the sample being investigated. Typically only Stokes photons are measured since these

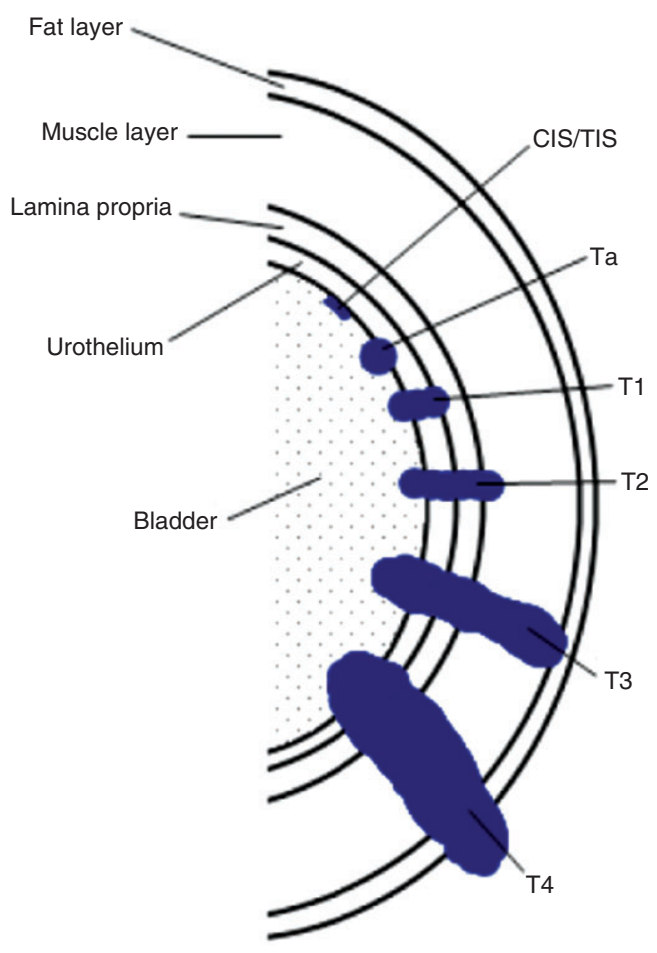

Figure 2 Staging of bladder tumors representing the location of each tumor with respect to the urothelium. have a higher intensity than Anti-Stokes photons. By recording and measuring the emission of inelastic photons in the form of Raman spectra, it is possible to detect the molecular differences between normal and diseased or cancerous cells within a biological sample, making it an ideal technique for diagnostics.

Raman spectroscopy is suitable for use in biofluid analysis because water is a weak Raman scatterer. The peaks of the different wavelengths that are emitted are unique to a particular molecule and this enables fingerprint-like recognition of complex molecular combinations. A Raman spectrometer can be fitted to a microscope so that a spectrum can be obtained from microscopic points in a sample, e.g., the nucleus of a cell. It has been shown that recording spectra from tissue and cells from patients with known disease, allows for the training of statistical classification algorithms that enables the diagnosis of disease from unknown samples. Various statistical techniques of unsupervised and semisupervized clustering have been employed to analyze Raman spectral alterations in disease conditions, and have been so far impressively successful in differentiating normal versus cancerous tissue.

There are various techniques used for applying Raman spectroscopy to biological samples. Most often, conventional Raman microspectroscopy is applied for the interrogation of biological material, but other techniques such as the use of a fiber-optic probe (for in vivo applications), Raman tweezers - i.e., the application of Raman spectroscopy in a laser tweezers set-up, Raman mapping - i.e., where a two-dimensional (2-D) Raman spectroscopic grid is generated of the tissue area which provides biochemical images and Kerr-gated Raman spectroscopy (for tissue depth profiling), have been used for analyzing 

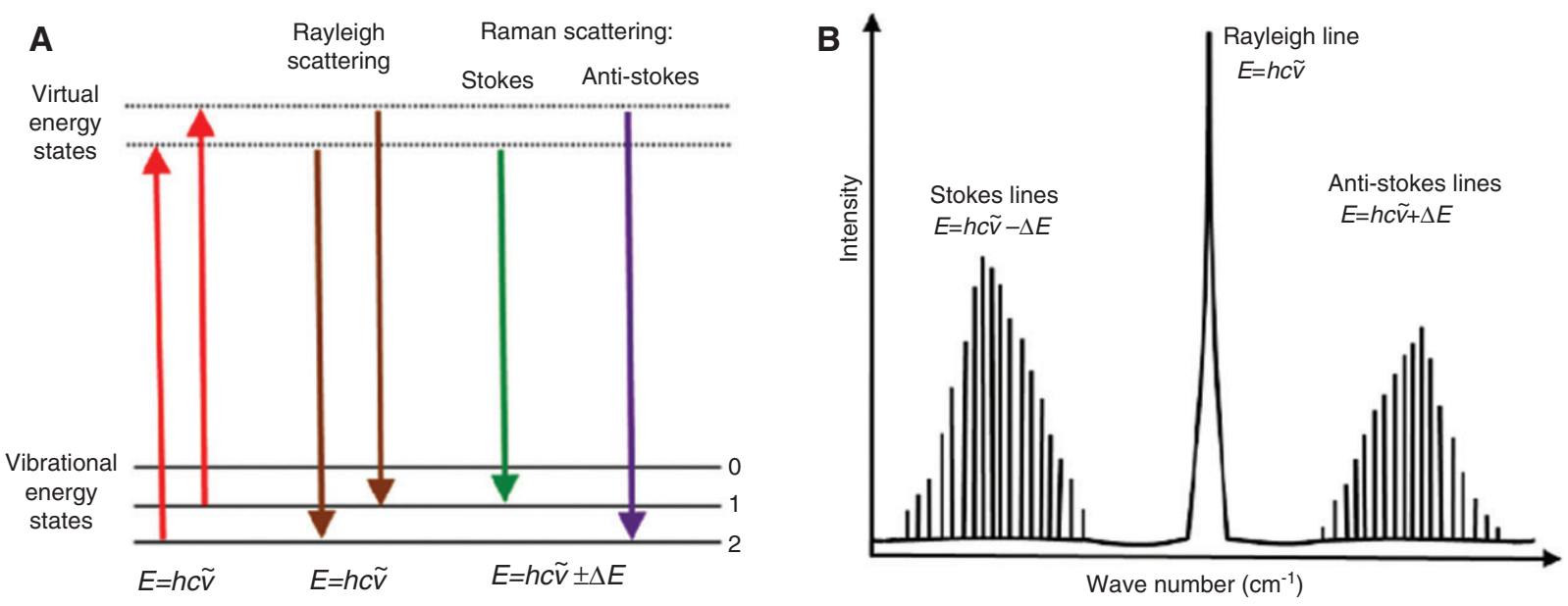

Figure 3 (A) Energy level diagram for incident photons, and photons emitted during Rayleigh scattering and Raman scattering. (B) Emission lines for Rayleigh scattered photons and Raman scattered photons. ( $E=$ energy, $h=$ Planck's constant, $c=s p e e d$ of light, $\tilde{v}=$ wavenumber of the photon, $\Delta E=$ change in energy).

the urinary bladder. Further details of the set-up for each method will be discussed in Sections 4.3 and 4.4.

Raman spectroscopy provides many advantages over other diagnostic techniques; it is fast, giving accurate diagnostic results in real time, but there are also limitations to this technique that need to be considered. Strong background signals are often detected which overlap with the Raman signal, making it difficult to distinguish which signals are associated with the biological sample. A variety of pre-processing statistical techniques have been applied to date to remove this noise, and a range of substrates and laser wavelengths have also been used to minimize background signals.

Good substrates for Raman spectroscopy should be transparent in the visible/near-infrared (NIR) region, produce a low background signal, and should be biocompatible and non-toxic for the cells or tissues placed on them. For example, glass in particular is known to produce a large background signal when used in conjunction with a NIR laser source, so the use of other optically transparent substrates such as calcium fluoride $\left(\mathrm{CaF}_{2}\right)$ and quartz are preferred for Raman spectroscopy in the NIR. A more detailed discussion on pre-processing is given in Section 3.5.

A basic Raman spectroscopic set-up must consist of four main components: (i) an excitation source, i.e. laser, (ii) an optical system to deliver the light to the sample with minimum power loss, (iii) a collection system with highly efficient optics that can collect an optimum amount of Raman scattered light whilst being able to remove all Rayleigh signals, and (iv) a detection system, i.e. spectrograph and charge-coupled device (CCD). A basic design of this optical method is shown in Figure 4, where additional optical elements can be inserted to optimize the system. There are many advantages to using lasers from the NIR range in the use of Raman spectroscopy for biological analysis; it allows for exploitation of the optical window which results in greater scattering within the biological sample, thus producing a larger Raman signal, whilst maintaining low levels of biological damage. Since the scattered Raman signal is very weak relative to the laser source beam, it is important to have efficient optical systems for both delivering the laser beam directly to the sample with sufficient power and for collecting the scattered Raman photons. To achieve this, a series of mirrors and lenses are used to direct the optical path directly to where it is needed, and filters are used to remove unwanted signals. It is also possible to

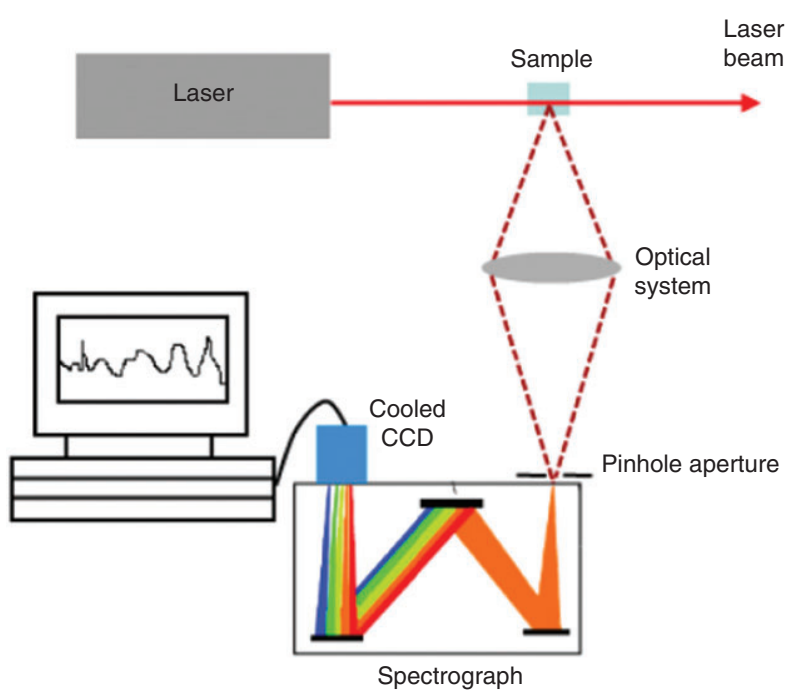

Figure 4 Basic set-up used for Raman spectroscopy. 
include a pinhole aperture to isolate the spectrum from a particular location in the sample, the effect of which is to greatly reduce the background signal and to enable point-topoint spectral mapping. The detection system consists of a spectrograph and a CCD detector. Thermal energy can often generate noise and dark current on the CCD which can block out the distinct Raman peaks. To avoid this, and to maintain a good signal-to-noise ratio $(\mathrm{S} / \mathrm{N})$, the $\mathrm{CCD}$ must be cooled.

\subsection{Conventional Raman microspectroscopy}

Conventional Raman microspectroscopy, involves the use of a microscope and a confocal aperture in order to isolate the Raman spectrum from a specific microscopic three-dimensional (3-D) point in a specimen and has been applied to both bladder tissue and cells for analysis and classification. The laser beam can be delivered at $180^{\circ}$ to the sample surface or at $90^{\circ}$, in either a reflection- or transmission-based system. Biological samples can be measured both in vitro and ex vivo with this method by mounting them onto a substrate. A Raman spectrometer can be fitted to an existing microscope so that a spectrum can be obtained from microscopic points in a sample, e.g., the nucleus of a cell, and this has been the basis of much work in bladder cancer diagnostics. Figure 5 illustrates the Raman microspectrometer that has been custom built in our lab for experiments in bladder cancer diagnosis. This basic design can be found in most commercial Raman microspectrometers from long established companies such as Renishaw and Horiba Raman. A state of the art Raman microscope will cost in the order of $\$ 100,000$ but an equivalent one can be built from basic elements for less than half this amount.
The design in our lab is as follows; a laser with a narrow bandwidth (Sacher Lasertechnik, Germany; TEC $5207080-100$, wavelength $785 \mathrm{~nm}$, power $120 \mathrm{~mW}$ ) is first passed through an optical isolator OI (Sacher Lasertechnik, Germany; ISO-35-0780) which prevents back reflections from the set-up from returning into the laser cavity and thereby damaging the laser head. This is followed by a line pass filter $L P$ (Semrock Inc., NY, USA; LL01 785-12.5) which removes any spurious lines from the laser that may result from additional modes being present. Only the line at $785 \mathrm{~nm}$ is allowed to reach the neutral density filter ND (Edmund Optics Inc., NJ, USA) which can be rotated in order to vary the power of the laser beam that is allowed to pass through it. Two lenses, $L 1$ and $L 2$ (Thorlabs, Germany), are used to expand the laser beam such that the diameter of the collimated beam is approximately equal to the back aperture of the microscope objective $M O$ (Olympus, Japan; $50 \times$, numerical aperture $\mathrm{NA}=0.85$, UMPlanFl) which will be discussed shortly.

A system of two mirrors $M$ is used to direct the collimated beam onto a dichroic beam splitter $D B$ (Semrock Inc., NY, USA; LPD01-785RS) which will reflect light at $785 \mathrm{~nm}$ but transmit other wavelengths. The dichroic beam splitter reflects the beam towards a hot mirror $H M$ (Edmund Optics Inc., NJ, USA), which is another form of dichroic beam splitter, in this case reflecting all wavelengths above $650 \mathrm{~nm}$ and transmitting all shorter wavelengths. The hot mirror is located within the fluorescence cube cavity of an inverted Nikon phase contrast microscope (Nikon, Toyko, Japan; Diaphot 300) and is carefully aligned such that it reflects the beam directly into the back of the microscope objective. The microscope objective is chosen such that it has a high numerical aperture which is essential to recover as many Raman backscattered photons

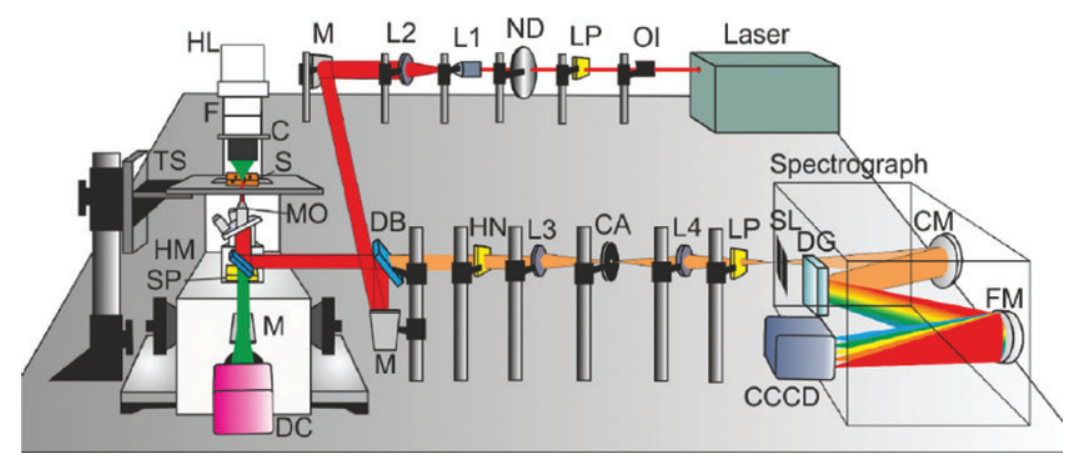

Figure 5 Schematic of a conventional Raman spectroscopy set-up.

OI, optical isolator; LP, line pass filter; ND, neutral density filter; L1 and L2, lenses; M, mirrors; DB, dichroic beam splitter; MO, microscope objective; HM, hot mirror; S, sample; TS, translation stage; HN, holographic notch filter; L3, lens; CA, confocal aperture; L4, lens; LP, long pass filter; SL, entrance slit; CM, collimating mirror; DG, diffraction grating; FM, focusing mirror; CCCD, cooled CCD camera; HL, halogen lamp; F, green filter; C, condenser; SP, short pass filter; DC, digital camera. 
as possible. It is also essential that the microscope objective efficiently transmits light in the NIR as this will also impact on the system to recover a strong Raman signal. Plan fluorite microscope objectives provide satisfactory performance. The microscope objective focuses the beam to a diffraction limited spot on the sample $S$. The sample is placed on a translation stage TS (Applied Scientific Instrumentation, OR, USA; LS-2000 and LS-50) that enables sample positioning relative to the laser spot. In the case of Raman diagnostics, it is desirable to position the cell such that the spot is located within the cell nucleus as this results in optimum diagnostic sensitivity.

The backscattered Raman photons (785-1100 nm) travel back through the microscope objective and are reflected by the hot mirror and then pass through the dichroic beam splitter. A holographic notch filter $H N$ (Kaiser Optical Systems Inc., MI, USA; HNF-785.0-1.0) serves to remove light at $785 \mathrm{~nm}$ that has made its way through the dichroic beam splitter, and to transmit all other wavelengths. Following this a third lens $L 3$ (Thorlabs, Germany) focuses the light to a $100 \mu \mathrm{m}$ confocal aperture $C A$ (Thorlabs, Germany). The presence of the confocal aperture ensures that light reaching the spectrograph has originated from a 3-D volume within the object in the order of $1 \mu \mathrm{m}$ in all three dimensions. This allows for 3-D localization of the Raman signal from the sample. A further lens $L 4$ (Thorlabs, Germany) forms a one-lens imaging system from the confocal aperture to the entrance slit $S L$ of the spectrograph (Andor Technology, Belfast, UK; Shamrock 500). A long pass filter $L P$ (Semrock Inc., NY, USA; LP02-785RU-25) is placed near the entrance slit in order to further reduce any Rayleigh scattered/laser light that has reached the spectrograph. Via a collimating mirror $C M$, a diffraction grating $D G$, and a focusing mirror $F M$, the image of the slit is projected onto a cooled CCD camera CCCD (Andor Technology, Belfast, UK; DU420A-BR-DD), where each row of pixels records an image of the slit corresponding to a specific wavelength, and in this way the Raman spectrum is recorded using the Andor Solis software system. It is essential to use a high quantum-efficiency, low-noise, cooled CCD in order to recover the weak Raman signal with minimal noise.

Independently, light from the 100-W halogen lamp $H L$ (Nikon, Japan) at the top of the inverted microscope passes through a green filter $F$ (Nikon, Japan) and then through an Abbe condenser $C$ (Nikon, Japan) which focuses the light onto the sample and into the microscope objective. Since the green light has a shorter wavelength than $650 \mathrm{~nm}$, the light passes through the hot mirror and then through the short pass filter SP (Semrock Inc., NY, USA; FF01-775/
SP-25), which is designed to transmit all wavelengths shorter than $775 \mathrm{~nm}$ and block higher wavelengths. This has the effect of preventing any further transmission of Rayleigh light that has managed to pass through the holographic notch filter. The green light passes into the body of the microscope where it reflects from an internal mirror through an internal imaging system, and images onto a digital camera $D C$ (Basler, Ahrensburg, Germany; acA2000-340 km) fixed to the front exit port of the microscope. Figure 6A shows an image of bladder cancer cells recorded on the digital camera, and Figure 6B the Raman spectrum recorded on the cooled CCD corresponding to the point indicated in Figure 6A. It is of note, that the wavenumbers $\left(\mathrm{cm}^{-1}\right)$ used on the axis can be converted into wavelength $(\mathrm{nm})$.

To perform a diagnostic measurement, spectra are recorded from multiple cells from each patient sample. Using a set of known samples, the spectra are grouped into categories according to pathological information
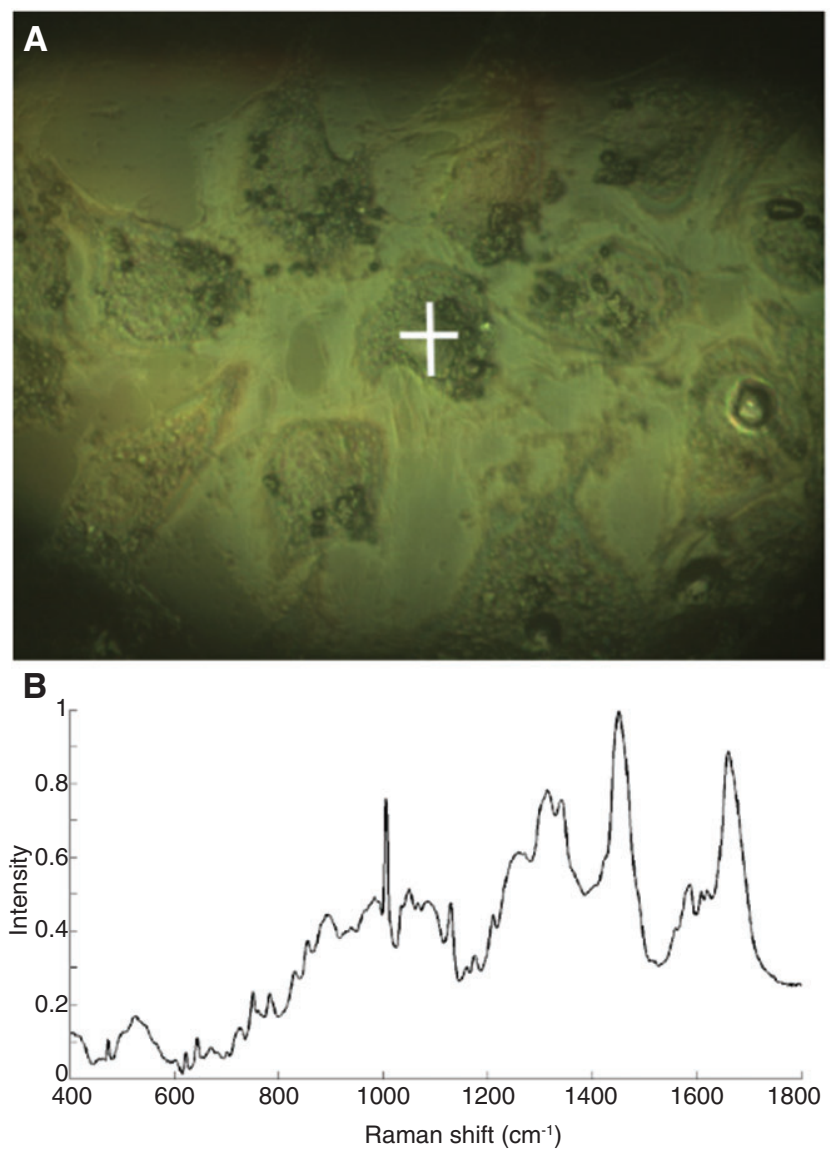

Figure 6 (A) An image of a fixed bladder cancer cell (T24, American Type Culture Collection) recorded on the digital camera, and (B) the Raman spectrum recorded on the cooled CCD corresponding to the point indicated in $(A)$. 
obtained from a consultant histopathologist. Application of the dimension reduction method of principal components analysis (PCA) enables training of a classification algorithm in order to identify the presence (and grade) of TCC, and the sensitivity/specificity of the approach is often determined using a cross validation method. A more detailed discussion of Raman-based diagnostics is given in Section 4. Another related approach is Raman mapping where the translation stage is scanned in three dimensions and sequential confocal Raman spectra are recorded. Statistical clustering approaches can be used to group points that share a similar biochemistry and these regions can be represented with pixels of the same color. Raman mapping is discussed in Section 5.

\subsection{Raman spectroscopy with a fiber-optic probe}

A fiber-optic Raman spectroscopy set-up consists of a specialized fiber probe, an excitation source (laser), fiber couplers to deliver the light in and out of the fiber, filters to remove unwanted signals and a detection system (spectrograph and cooled CCD camera), as can be seen in Figure 7. This fiber-optic probe can be inserted during a cystoscopy procedure, and be used to give real-time analysis and classifications of the urothelium. Similar to conventional Raman microspectroscopy, the Raman fiber-optic probe can be used to monitor in vitro and ex vivo specimen also. The main advantages of this system is the potential to replace the need for multiple biopsies, a reduction in the turn-around time for diagnosis, a reduction in histological/pathological costs and an improved surgical procedure by using the probe to identify border regions of diseased or damaged tissue.

A Raman probe for cystoscopic or endoscopic diagnostics must be able to fit into the instrument channel of a standard cystoscope (or endoscope or catheter), be biocompatible and robust enough to withstand decontamination/disinfection processes. NIR lasers $(785-830 \mathrm{~nm})$ are generally used for Raman fiber-optic probes because they minimize thermal damage to the tissue sample being analyzed. It is also essential that there is a low Raman signal generated within the probe. The general design of a Raman fiber-optic probe is shown in Figure 7; consisting of a central delivery fiber which is surrounded by several collection fibers, where the diameter of each fiber is between 100 and $400 \mu \mathrm{m}$ depending on the system design. Typically a bandpass filter is placed at the tip of the excitation fiber to remove unwanted background signals, and a longpass filter is placed at the tip of the collection fibers to prevent Rayleigh scattered light from entering these fibers. Lenses (such as a ball lens, made from sapphire) at the probe tip or tapered fiber tips can also be used to improve the efficiency of delivering both the excitation light to the tissue and Raman scattered light into the collection fibers (further information is available in [24]).

Commercially available Raman probes have been applied to the urinary bladder for diagnostics, such as the Enviva Biomedical Raman Probe (Visionex Inc., Atlanta, GA, USA) and the Emvision Raman probe (Emvision, LLC, Loxahatchee, FL, USA). Many homemade Raman probes have also been designed that could be integrated into in vivo bladder studies. An optical multi-fiber catheter was designed by de Lima et al. [25], with greater efficiency, lower background signals and greater flexibility

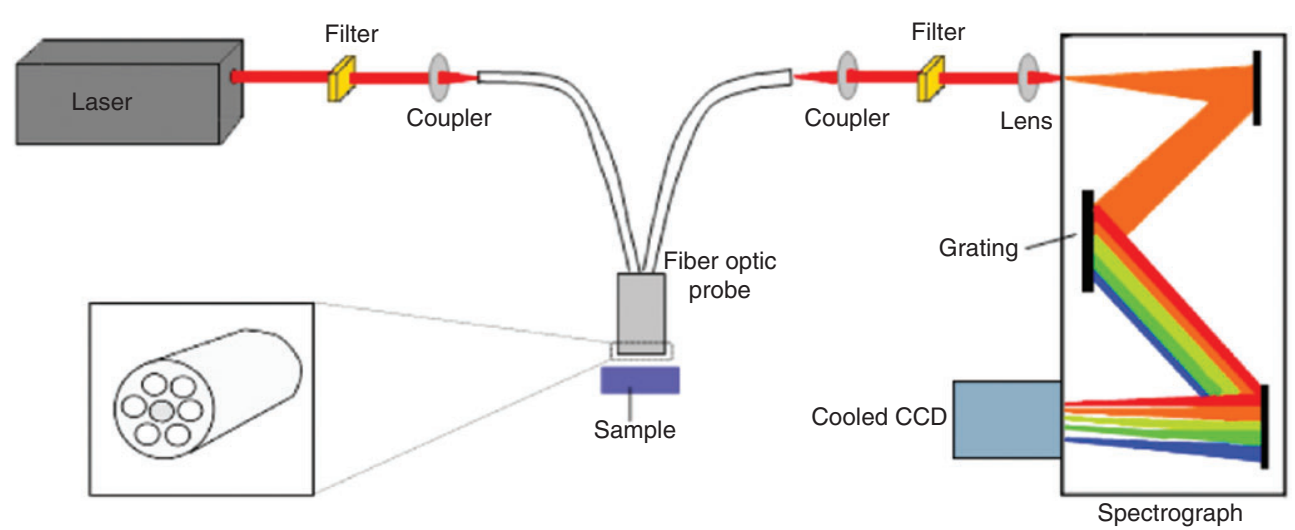

Figure 7 Schematic of the Raman fiber-optic probe set-up, which can be applied to biological samples both in vivo and in vitro, with a front view of the design of a fiber-optic probe inset, showing the central delivery fiber which is surrounded by several collection fibers. It is of note that it is often more conventional to integrate the filters into the tip of the fiber-optic probe instead, but for ease of illustration, they have been shown in the positions above. 
than many other fiber probes. This was achieved by using a low NA (0.12) excitation fiber and a higher NA (0.22) collection fiber, and replacing the standard silver epoxy resin adhesive and transparent tube found on catheters with a black epoxy resin adhesive and a black Teflon tube covering. A confocal Raman probe was recently constructed by Barman et al. [9] by introducing a $150 \mu \mathrm{m}$ pinhole aperture into the system at the proximal end of the fiber probe, which the authors stated to provide higher specificity than a standard Raman probe. In another recent study, a subcutaneous Raman needle probe was designed by Day et al. [26] incorporated a miniature fiber probe into a standard hypodermic needle; this was achieved by using only two fibers, one for excitation and one for collection, with laser power of $24 \mathrm{~mW}$ reaching the tissue sample and an acquisition time of $30 \mathrm{~s}$ for each measurement.

One of the main problems associated with Raman fiber-optic probes is that the fused silica from the fiber generates a Raman signal which can block out the signals from the biological sample. This can be reduced using low OH fibers. An alternative option, suggested by Koljenović et al. [27], is to record spectra from the high wavenumber range (2400-3800 $\mathrm{cm}^{-1}$ ) where there is no Raman signal from the silica fiber. However, the fingerprint region $\left(400-1800 \mathrm{~cm}^{-1}\right)$ is typically used for analyzing biological specimen since this is where the optimum Raman signals are collected. It is of note, that Praveen et al. [28] were recently able to reduce fluorescent background signals by integrating a modulated NIR laser into the fiber-optic Raman system. Other difficulties with Raman probes are the long acquisition times needed, poor $\mathrm{S} / \mathrm{N}$, the lack of wide-field measurement capabilities, and complications with designing a probe which can withstand everyday usage in a clinical environment [6].

A potential further development for Raman fiber-optic probes for diagnostics is based on photonic crystal fibers since there are many advantages to using these instead of silica fibers. Photonic crystal fibers are hollow, and therefore don't add any contributions to the Raman signals collected [29]. It is also possible to use one photonic crystal fiber for both excitation and collection, thus overcoming the technical challenge of packing several fibers into a small probe.

\subsection{Raman optical tweezers}

A Raman tweezers is based on combining the principle of Raman microspectroscopy as described previously and optical trapping (or laser tweezing) [30, 31]. The latter is a technique that utilizes a high NA microscope objective, usually configured in an inverted microscope, together with a suitable laser, to trap a cell in the waist of a focused laser beam. The tightly focused light creates a sharp gradient of intensity which leads to gradient forces trapping the cell. The trapped cell can be suspended in a liquid (or air) environment and moved in three dimensions by manipulating a component in the optical set-up. By combining Raman microspectroscopy with optical trapping, individual cells can be biochemically probed under physiological conditions or in microfluidic chips.

There are at least two separate physical models that describe optical trapping. The first of these is based on Mie scattering, i.e., on the condition that the trapped particle has a diameter that is significantly greater than the
A

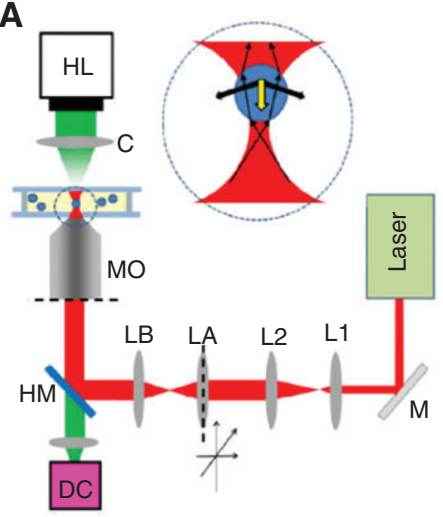

B

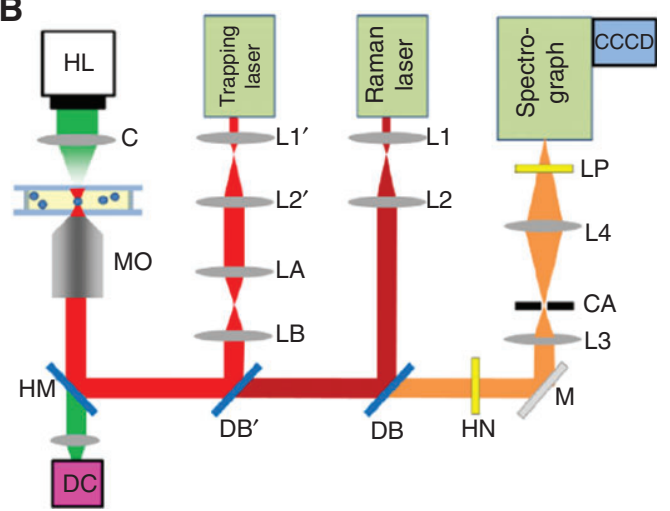

Figure 8 Schematic of a standard Raman optical tweezers set-up (A) and a dual beam Raman tweezers set-up (B).

$\mathrm{HL}$, halogen lamp; C, condenser; MO, microscope objective; HM, hot mirror or more accurately a suitably flat dichroic mirror; DC, digital camera; L, lenses; M, mirror; DB' and DB, dichroic beam splitter; HN, holographic notch filter; CA, confocal aperture; LP, long pass filter; CCCD, cooled CCD camera. 
wavelength of the light. In this model basic ray optics can be used to determine the gradient optical forces acting on the particle. An illustration of this model is shown in Figure 8A. Here two rays of light can be seen intersecting at the focal point of the microscope objective. At the surface of a sphere they are refracted due to a difference in the refractive indices of the chamber fluid and the sphere. This refraction brings about a change in momentum of the light rays, which according to Newton's third law must be transferred to the sphere, illustrated by the thick black arrows in the figure. The resultant forces on the sphere act to trap the particle in a static position in the horizontal plane, while the sum of their components in the vertical direction force the particle back towards the focal point. In addition to the gradient office there is a second force acting on the particle known as the scattering component which can be described as a "fire hose" pushing the sphere away from the microscope objective in the direction of propagation [31]. So long as the gradient force is greater than the scattering force, the sphere comes to rest at a point of equilibrium, slightly "downwind" of the lens' focal point, where all of the forces cancel. In instances where this is not accomplished a second counter-propagating trap can be implemented using a second microscope objective [32].

The second physical model that describes optical trapping is based on Rayleigh scattering where the particles are significantly smaller than the wavelength of the light, which is derived using the theory of electromagnetics. For cellular analysis, where the cell size is approximately 5-10 times that of the wavelength, trapping operates somewhere in between the two models. Regardless of which model is valid, the strength of the trap is proportional to the laser power and so high-power lasers are preferable, which necessitates longer wavelengths (e.g. $1064 \mathrm{~nm}$ ) to minimize photobleaching and cell damage. Another important property is the laser beam profile, which should have a clean Gaussian shape (known as a TEM00 spatial mode). This property ensures the narrowest possible focus and balanced trapping forces acting on all sides of the cell. Other desirable properties include good pointing stability and low power fluctuation.

A typical set-up for optical trapping is shown in Figure 8A. A laser is expanded using two lenses $L 1$ and $L 2$ which should provide sufficient magnification such that the laser beam is expanded to have a diameter approximately equal to the aperture at the back of microscope objective $M O$; ideally the $1 / \mathrm{e}^{2}$ intensity points on the beam profile should match the aperture which ensures that $87 \%$ of the energy will enter the objective. The expanded laser beam then passes through two identical lenses, $L A$ and $L B$, which are separated by a distance equal to twice their focal length. The purpose of these two lenses is to provide a means of moving the focal point of the trap in the $\mathrm{x}, \mathrm{y}$ and $\mathrm{z}$ dimensions.

The distances in the set-up should be arranged such that $L B$ forms a one-lens imaging system between the plane of $L A$ and the back of the microscope objective (both planes are marked with dashed lines in the figure). This property ensures that if the lens, $L A$, is physically moved horizontally and vertically the beam will still target the microscope objective aperture exactly, while the angle of the beam will change resulting in a shift of the position of the trap. The microscope objective must have a sufficiently high NA (>1.25) to create a gradient that is capable of trapping and so oil immersion objectives are usually used. It must also have high quality aberration compensation to create a tightly focused trap. Located between the lens $L B$ and the microscope objective is a dichroic beam splitter of some variety, similar to the previous hot mirror $H M$, but with sufficient surface flatness to ensure the laser beam is unaffected, which reflects the trapping laser while allowing the shorter wavelength light from a halogen lamp $H L$ pass through unaffected. Light from the halogen lamp illuminates the sample via a condenser lens $C$ and forms a magnified image on a digital camera $D C$ so that the trapped particle can be observed. The trap can be moved in three dimensions by moving the lens $L A$ correspondingly. The trapped particles are usually contained in liquid within a glass chamber, the base of which has a thickness equal to that of a standard coverslip $(170 \mu \mathrm{m})$. A more detailed discussion of optical trap design can be found in [31].

A direct comparison of the Raman microspectroscopy set-up shown in Figure 5 (not considering the elements to the right side of the dichroic beam splitter $D B$ ) and the optical tweezers set-up shown in Figure 8A leads to the conclusion that both systems have similar characteristics. It is clear that the Raman system is effectively the same as the tweezers system except for the presence of lenses $L A$ and $L B$. These could easily be included or left out if trap movement is not needed. Therefore, by ensuring that the laser and the microscope objective have the properties necessary for trapping as discussed above, then the Raman microspectroscopy system can also serve as an optical trap.

Interestingly, it has been shown that cells trapped in such a system are trapped by the nucleus [33] and this ensures that the Raman signal measured essentially emanates from the nucleus of the cell which is advantageous for diagnostic classification. In such a case it may be possible to remove the confocal aperture in the system and thereby increase the power in the Raman signal by obtaining an average spectrum from the entire nucleus.

Another compromise is to use a dual beam Raman tweezers such as that described in [34]. Such a system, 
as illustrated in Figure $8 \mathrm{~B}$ combines the optical trap in Figure 8A and the Raman microspectrometer in Figure 5. In this case two lasers are employed: one for Raman excitation (expanded using $L 1$ and $L 2$ ) and one for trapping (expanded using $L 1^{\prime}$ and $L 2^{\prime}$ ). The only element that was not already introduced is the dichroic beamsplitter, $D B^{\prime}$, which reflects the $1064 \mathrm{~nm}$ wavelength but transmits all others. It is of note, that for the sake of brevity some of the optical elements shown in Figure 5 were omitted.

There are a number of advantages to such a decoupled system; firstly it allows for the use of a $1064 \mathrm{~nm}$ laser to trap the cell thereby minimizing cell damage, and facilitating lower wavelengths to be used for Raman excitation. Secondly, it allows for probing of different regions in the trapped cell for isolated confocal Raman spectra. This can be achieved by moving the trap in 3-D around the confocal spot of the Raman microspectrometer.

Raman tweezers offer exciting new avenues of research, particularly in the areas of microfluidics and urine cytology. This technique can be used for investigating the biochemistry of living cells in their natural environment with 3-D spatial resolution [33]. It can also be used for diagnostic classification for bladder cells. Both bladder and prostate cells have been investigated in this way in the literature [32, 35-37] and some of the details of this research is discussed later in Section 4.4.

\subsection{Pre-processing of Raman spectra}

Each Raman spectrum consists of three main components: Raman signals, the baseline and noise. The presence of these unwanted background/baseline signals and noise is often reduced using pre-processing techniques before the classification process as they can lead to a larger diagnostic error otherwise. Numerous techniques have been applied to achieve this:

- Wavenumber calibration: Small changes in the optical set-up, and/or a change in temperature can result in a shift in the Raman spectrum along the wavenumber axis. Accurate calibration is therefore required to allow for comparison of Raman data obtained from different Raman spectroscopic set-ups. This is particularly important when creating standard medical diagnostic databases for disease identification. The purpose of wavenumber calibration is to assign a particular wavenumber shift to each row of pixels in the cooled CCD camera [38]. To perform wavenumber calibration on a Raman spectroscopic set-up, a known sample such as silicon is measured, and the positions obtained using the Raman system are calibrated with the precisely known Raman peak positions for the calibration sample used.

- Baseline correction: The baseline of a spectrum is described as the slowly varying curve seen in the lower range of the spectrum without the distinct Raman peaks. This baseline can be varying or sloped, and is usually due to unwanted background signals or fluorescence. To remove this background contribution, baseline correction is performed by subtracting a linear or polynomial fit of the baseline from the original spectrum. Alternatively, the background signal can be recorded independently and subtracted from the cell/tissue spectrum. However this can be difficult as the overall intensity can vary significantly between captures. Further information about baseline correction choices can be found elsewhere [39].

- Normalization: Normalization in the intensity axis is performed to adjust peak intensity values from each spectrum in order to provide a common scale for comparing Raman peaks across a range of spectra. Normalization is achieved by dividing each variable in a spectrum by some constant. There are two main methods of obtaining this constant value depending on whether peak normalization or vector normalization is being used. For peak normalization, the constant is measured as the height difference between the baseline and the maximum point of a chosen peak. Vector normalization obtains the constant value by calculating the sum of the intensity values for each variable in the spectrum, and finding the square root of this value [40].

- Extended multiplicative scatter correction: This is a model-based method for separating physical and chemical variances within spectra, as explained elsewhere $[40,41]$. This method has the ability to separate Raman-scattered signals from background chemical absorbance signals. This method of pre-processing allows for better interpretation of Raman spectra since background chemical signals are removed and only Raman-scattered signals remain.

- Wavelength modulation: Modulated Raman spectroscopy can be used to extract Raman signals from the background. This is achieved by modulating the excitation wavelength which results in a shift of the Raman signals while the unwanted fluorescence background remains static. By applying statistical methods such as PCA it is possible to obtain spectra which are free from background contributions [13, 42]. 


\section{Cancer diagnostics using Raman spectroscopy}

In the last 10 years, there has been growing interest into the diagnostic potential of Raman spectroscopy for classifying healthy and cancerous bladder tissue, as well as identifying the grade (or stage) of cancer present. This approach generally involves the training of a statistical algorithm based on known pathological samples, and as such, some degree of human error is introduced into the method from the beginning. Nonetheless, Raman-based bladder diagnostics has shown increasingly impressive results, and the latest studies have demonstrated the potential of in vivo real-time clinical diagnosis using a fiber-optic probe. It has also been shown that Raman spectroscopy can be utilized for diagnosing cells obtained from voided urine, thus becoming a completely non-invasive method of accurately detecting bladder cancer. In this section, the progression in the area over the past 10 years is reviewed and the sensitivities and specificities that have been achieved to date under various experimental settings are highlighted.

\subsection{Diagnostic algorithms}

To identify the pathology associated with each set of Raman data, the spectra obtained are initially subjected to pre-processing methods before undergoing further multivariate statistical analysis. This section provides information on the different multivariate techniques that have been used to develop diagnostic algorithms for classification of data obtained from Raman spectroscopy. Techniques such as PCA and linear discriminant analysis (LDA) are most commonly employed for classification of bladder disease, but other techniques have also been demonstrated which will be further explained as follows:

- Principal components analysis: PCA is a powerful statistical tool used to reduce the number of variables within a data set. To do this, PCA transforms the spectral data into a set of variables called principal components (PCs), whereby all PCs are orthogonal to each other and they are generated in such a way as to represent as much variance within the dataset as possible. Computationally, PCs are found by determining the covariance matrix of the data set, and calculating the eigenvectors and eigenvalues of this covariance matrix. The first PC is the linear combination of the variables and the eigenvector corresponding to the largest eigenvalue. The eigenvector with the second largest eigenvalue is used to construct the second PC, and so on. Thus, further PCs are orthogonal to the previous ones, whilst describing the maximum variance remaining in the data. By leaving out PCs with corresponding eigenvalues that are close to zero, it is possible to significantly reduce the dimensionality of the data whilst maintaining almost all of the spectral information; for example, in an average Raman spectral measurement data is collected between $400-1800 \mathrm{~cm}^{-1}$, with measurements taken approximately every $10 \mathrm{~cm}^{-1}$, thus providing $\sim 140$ variables in the original data set, which can be reduced to $\sim 10$ using PCA. Further analysis can then be applied to these PCs to organize them into groups, or clusters, representing different tissue pathologies; techniques such as linear discriminant analysis (LDA) or logistic regression analysis (LRA) are often utilized. The main advantage of this tool is that it provides a simpler representation of the data and allows for faster classification algorithms to be designed.

- Linear discriminant analysis: LDA, also known as Fisher's discriminant analysis, is a supervised multivariate technique used to optimize class separability by finding the direction that provides the best separation for two or more groups of data. LDA is often applied to PC scores to further reduce the dimensionality of the data set. This is achieved by finding a linear combination of vectors that maximize the ratio of between-group variance and within-group variance [38]. By maximizing this ratio, LDA is able to provide the optimum separation for each group, thus improving classification results.

- Cluster analysis: Cluster analysis refers to unsupervised statistical techniques used to organize the spectra into clusters sharing similar biochemistry. Most clustering methods are based on finding distances between and within each cluster. An average spectrum can be used to represent each cluster and these cluster averaged spectra (CAS) can be further classified into distinct groups such as cancerous or non-cancerous. For supervised classification methods, such as LDA or LRA, the group membership of all the spectra is known and this information is used to help reveal the structure of the data which then allows the classification of future observations. For unsupervised methods, such as hierarchical cluster analysis (HCA) or K-means clustering, the class membership is unknown and the aim is to group similar observations together. Cluster analysis can be applied to the spectral data set of a Raman map transformed by PCA. This allows for each grid element 
of the Raman map to be assigned to a cluster of tissue area with similar molecular composition.

- Cross validation: Cross validation is often used to estimate how accurately the diagnostic model will perform. This is achieved by assessing the results of the statistical algorithm when applied to a validation set of data. The most common method used for assessing Raman spectra models is leave-one-out cross validation. Leave-one-out is based on using a single spectrum as the validation set, and the remaining spectra are used as the training set for the algorithm. This is repeated to test each spectrum in the dataset, and can be used to determine how accurate the model is at predicting the pathological status of the sample. Blind testing and double blind testing can also be applied, these are based on concealing pathological information from the data in order to remove observer bias.

\subsection{Application of Raman spectroscopy to bladder biopsy/tissue samples}

In this section the specific studies relating to the application of Raman spectroscopy to biopsy and tissue samples for the purpose of bladder disease diagnosis are reviewed. This paper attempts to extrapolate the best practice in terms of the methods and tools used. This section begins by discussing the sources of tissue and the manner in which they can be stored and prepared for Raman spectroscopic analysis. This is followed by a review of each of the major studies in the area, the techniques used and the resulting sensitivities and specificities that were achieved.

To diagnose bladder tissue using conventional Raman spectroscopy, tissue samples can be removed from tumorous regions during transurethral resection surgical procedures, such as transurethral resection of bladder tumor (TURBT) or transurethral resection of the prostate (TURP). Both of these are minimally invasive surgical procedures, carried out via the urethra by passing surgical tools through a cystoscope into the bladder. Large tumors or CIS are more difficult to remove or analyze during TURBT or TURP, so biopsy samples can be taken during cystoscopy procedures instead. A more invasive surgical procedure for the removal of all or part of the bladder is known as a cystectomy, and these tissue samples can also be diagnosed using Raman spectroscopy.

Raman spectroscopy can be effectively applied to both fresh and preserved bladder tissue samples. There are numerous methods of preserving bladder tissues so that they can be stored for long periods of time without largely affecting their natural biochemistry. Tissue samples can be snap-frozen with liquid nitrogen upon removal from the patient, and then stored at $-80^{\circ} \mathrm{C}$. The main advantage with freezing is that the biochemistry of the samples are not altered and they can be analyzed simply by returning the samples to room temperature. Bladder tissue could also be stored in paraffin-wax blocks; however it is more difficult to analyze tissue samples that have been stored in paraffin with Raman spectroscopy since paraffin has large distinct peaks that appear in the Raman spectra. Despite this, it is possible to restore the tissue to a usable state with the use of particular deparaffinization techniques.

Prior to in vitro/ex vivo Raman spectral measurements, bladder tissue samples should be brought to room temperature, cut into thin sections (generally $<50 \mu \mathrm{m}$ thick) and placed onto a substrate such as $\mathrm{CaF}_{2}$, aluminum or quartz. Similarly, biopsy samples should be placed directly on to the substrate at room temperature.

These tissue samples are then inserted to the Raman set-up for measurement and analysis. Table 3 shows the Raman capturing methods, laser details, substrates used and the wavenumber range recorded in publications that have used conventional Raman microspectroscopy to diagnose and grade bladder cancer tissue and biopsy samples.

In 2002, in what is believed to be the first Ramanbased bladder cancer diagnostic study, Stone et al. [43] investigated the combined use of conventional Raman spectroscopy with a diagnostic algorithm to classify or

Table 3 Capture method, substrates and wavelengths used for Raman spectroscopy based diagnostic model for diseases associated with bladder tissue.

\begin{tabular}{lllrrrr}
\hline References & Year & $\begin{array}{l}\text { Raman optical } \\
\text { method }\end{array}$ & $\begin{array}{r}\text { Laser } \\
\text { wavelength }(\mathbf{n m})\end{array}$ & $\begin{array}{r}\text { Laser power [at } \\
\text { sample] }(\mathbf{m W})\end{array}$ & $\begin{array}{l}\text { Substrate (if } \\
\text { applicable) }\end{array}$ & $\begin{array}{r}\text { Wavenumber } \\
\text { range }\left(\mathbf{c m}^{-1}\right)\end{array}$ \\
\hline$[43]$ & 2002 & Conventional & 830 & 85 & Calcium fluoride & $400-1800$ \\
{$[44]$} & 2004 & Conventional & 830 & 300 & NS & $300-1800^{\text {a }}$ \\
{$[45]$} & 2006 & Mapping & 845 & 100 & Calcium fluoride & $400-1800$ \\
{$[11]$} & 2009 & Conventional & 830 & $300[100]$ & Calcium fluoride & NS \\
\hline
\end{tabular}

aExact wavenumber range not given, assumption made from Raman spectra provided; NS, not specified. 
distinguish between different pathological grades of bladder epithelial tissue. Bladder tissue samples were collected during cystoscopy procedures from 12 patients, and classified as being normal, CIS, LGC, medium-grade carcinoma (MGC) and HGC by histopathologists. At least 15 Raman spectra were obtained from each sample, with an integration time of $10 \mathrm{~s}$ per spectrum. Spectra were then put into a multivariate analysis consisting of PCA, LDA and leave-one-out cross validation, which separated the spectra into five distinct groups with sensitivities between $78 \%$ and $98 \%$ and specificities between $96 \%$ and $99 \%$. Further breakdown of these results can be seen in Table 4 . These results show excellent group separation, however some overlap is found between CIS and LGC, and between MGC and HGC which the author notes is either due to the biochemical continuum of disease or from difficulty in distinguishing between pathological groups during histopathological classification. Nonetheless, this paper clearly demonstrates the potential of Raman-based classification for improving the diagnosis of bladder pathology.

Members of the previous study further developed this diagnostic model using Raman spectroscopy to distinguish between a larger range of bladder tissue pathologies in vitro and to grade and stage TCC [44]. Conventional Raman spectroscopy was applied to a range of bladder tissue pathologies (normal, cystitis, CIS, G1 TCC, G2 TCC, G3 TCC, G3 squamous dysplasia and adenocarcinoma). These tissues were obtained from 72 patients during TURBT, TURP and cystectomy procedures, and samples were classified by a consultant uropathologist and a consultant pathologist. All tissues were snap-frozen and stored at $-80^{\circ} \mathrm{C}$, and then brought back to room temperature for measurement. Raman spectra obtained from these samples were analyzed using PCA and LDA based on a leave-one-out validation method. The results of an eight-group diagnostic algorithm are shown in Table 5. Seven- and three-group algorithms were also developed; all yielding sensitivities and specificities greater than $82 \%$ and $95 \%$ respectively, a further breakdown of these results can be seen in Table 5 . The authors mention that

Table 4 Sensitivities and specificities for distinguishing between different bladder pathology groups, derived by Stone et al. [43].

\begin{tabular}{lrr}
\hline Pathology & Sensitivity (\%) & Specificity (\%) \\
\hline Normal & 93 & 99 \\
CIS & 83 & 97 \\
Low-grade cancer & 96 & 96 \\
Medium-grade cancer & 78 & 98 \\
High-grade cancer & 98 & 96 \\
\hline
\end{tabular}

CIS, carcinoma in situ.
Table 5 Sensitivities and specificities achieved from algorithms generated by Crow et al. [44] for diagnosing the histological grade of bladder tissue.

\begin{tabular}{lrr}
\hline Histological grade & Sensitivity (\%) & Specificity (\%) \\
\hline Eight-group algorithm: & & \\
Normal & 95 & 98 \\
Cystitis & 87 & 98 \\
CIS & 84 & 99 \\
G1 & 71 & 95 \\
G2 & 78 & 94 \\
G3 & 92 & 98 \\
G3 squamous & 100 & 99 \\
G3 Adenocarcinoma & 92 & 100 \\
Seven-group algorithm: & & \\
Normal & 96 & 98 \\
Cystitis & 86 & 98 \\
CIS & 82 & 99 \\
G1/G2 & 93 & 99 \\
G3 & 93 & 99 \\
G3 squamous & 100 & 99 \\
G3 Adenocarcinoma & 92 & 100 \\
Three-group algorithm: & & 97 \\
Normal & 95 & 95 \\
Cystitis & 90 & 98 \\
TCC \& CIS & 91 & \\
\hline CIS Carcinomain & &
\end{tabular}

CIS, carcinoma in situ; G1, low grade; G2, medium grade; G3, high grade; TCC, transitional cell carcinoma.

the misclassification of G1/G2 TCC could be due to the biochemical continuum of the disease or because the samples are between G1 and G2 TCC. Further algorithms were generated based on staging of pathology (i.e. Ta, $\mathrm{T} 1, \mathrm{~T} 2)$ which also yielded high results, as shown in Table 6. These algorithms were based on the molecular composition of the tissue, not the actual degree of invasiveness. With this thorough study, Crow et al. [44] extended the use of Raman spectroscopy for the diagnosis of bladder pathology and experimentally demonstrated that both the grade and stage of the disease

Table 6 Sensitivities and specificities achieved from algorithms generated by Crow et al. [44] for diagnosing the pathological stage of bladder tissue.

\begin{tabular}{lrr}
\hline Pathological stage & Sensitivity (\%) & Specificity (\%) \\
\hline Three-group algorithm: & & \\
Ta & 95 & 96 \\
T1 & 91 & 94 \\
T2 & 80 & 98 \\
Two-group algorithm: & & \\
Ta & 96 & 96 \\
T1/T2 & 96 & 96 \\
\hline
\end{tabular}

Ta, T1, and T2, tumor states according to Table 2. 
could be identified using Raman classification with high sensitivities and specificities. This paper clearly shows the capabilities of Raman classification as a valuable tool for assisting the pathologist in order to improve the accuracy of diagnosis.

In 2006, de Jong and colleagues [45] used Raman spectroscopic mapping (mapping refers to biochemical images obtained using Raman microspectroscopy and is discussed in more detail in Section 5) to create a spectral model that could differentiate between tumor and nontumor bladder tissue in vitro. Fifteen snap-frozen bladder tissue samples were obtained consisting of normal (2), cystitis (3), T2-T3 (9) and TIS (1) which were later confirmed by a pathologist using hematoxylin and eosin (H\&E) staining. Spectra were obtained from each tissue sample in a 2-D grid like fashion, with a collection time of $20 \mathrm{~s}$, to create a 2-D Raman map of the area. All spectra were baseline-corrected, PCA was applied and the spectra were grouped into 90 separate clusters, where the spectra within each cluster showed a similar biochemical composition. Each cluster was assigned a color code, and in this way, images were created highlighting areas of similar biochemical composition. A CAS was then calculated for each cluster and classified as tumor or non-tumor in accordance with histopathology. The non-tumor group consisted of 37 CAS (representing collagen, muscle fibers and inflammation sites), and the tumor group consisted of 53 CAS (representing CIS, Ta, T1, T2 and T3). All 90 CAS were subjected to further statistical analysis (HCA) with Wards cluster algorithm and Euclidean distance measurements, and it was found that one cluster consisted solely of non-tumor CAS, whereas the other contained all 53 tumor CAS and 13 non-tumor CAS, all of which were from muscle and inflammation sites misclassified by the algorithm. The author comments that this misclassification might be due to the fact that these non-tumor CAS that were clustered with tumor tissues contained considerably less collagen which is often used to represent the presence of cancer. Therefore, to improve the accuracy of diagnosis, an LDA model was trained from these 90 CAS that was able to distinguish between tumor and non-tumor tissue with 100\% sensitivity and 95\% specificity. A second algorithm based on a leave-one-out cross validation model was also developed which resulted in a sensitivity of $94 \%$ and specificity of $92 \%$. This paper demonstrated the capability of Raman spectroscopic mapping with a diagnostic algorithm to discriminate between tumor and non-tumor bladder tissue with a high accuracy and emphasizes the potential of the technique to obtain color-coded images that could aid in precision surgery, should it be translated to an in vivo setting.
And finally, in 2009, Grimbergen et al. [11] investigated the potential of a combined diagnostic modality using fluorescence with conventional Raman spectroscopy to improve the diagnosis of bladder cancer. This was achieved by applying Raman spectroscopy to bladder biopsy samples with and without the presence of 5-ALA in vitro. 5-ALA is a photosensitive dye that leads to an accumulation of protoporphyrin IX (PpIX) in malignant cells and is often used in fluorescence cystoscopy to improve the detection of bladder cancers. Ninety-two biopsies were obtained from 73 patients without 5-ALA during TURBT and 38 biopsies with 5-ALA were obtained from 19 patients that underwent fluorescence-guided endoscopy, which were evaluated as normal, cystitis, CIS, G1 TCC, G2 TCC and G3 TCC by a histopathologist. Standard Raman spectra were measured from both biopsy groups, and preprocessed by applying baseline correction and normalization. Raman spectra from fluorescent and non-fluorescent groups showed agreement in some bands of significant difference with respect to normal and cystitis samples. An algorithm was derived to distinguish between cancer and non-cancerous tissue, using PCA and LDA, which was based on a leave-one-out cross validation of biopsies without 5-ALA, and when applied to non-5-ALA samples, a sensitivity of $88 \%$ and a specificity of $80.1 \%$ were achieved. However, when applied to the 5-ALA samples, a sensitivity of $42 \%$ and a specificity of $71.1 \%$ were found, indicating a change in the biochemistry of bladder tissue in the presence of 5-ALA. This resulted in the development of a second algorithm based on 5-ALA samples, which gave a sensitivity of $75.4 \%$ and specificity of $88.7 \%$ when applied to samples containing 5-ALA. And finally, a third algorithm was designed based on a combination of Raman spectroscopy and fluorescence prediction measurements that gave a sensitivity of $100 \%$ and specificity of $80.8 \%$ for distinguishing between cancerous and noncancerous biopsy samples containing 5-ALA. This combined modality of Raman spectroscopy with fluorescence would have an advantage for in vivo analysis of bladder tissue, whereby the author proposes to use 5-ALA-based fluorescence for guided resection of bladder cancer where 5-ALA fluorescence imaging would be used for large area screening, and the Raman spectroscopic algorithm would be used for accurate diagnosis at a specifically targeted region of interest.

\subsection{Raman cystoscopy}

Raman fiber-optic probes can be easily inserted during a cystoscopy procedure to give real-time analysis and 
classifications of the bladder urothelium. Similar to conventional Raman microspectroscopy, fiber-optic Raman probes can also be used to measure samples in vitro and ex vivo also. This section provides information on the progression of fiber-optic Raman probes applied to the urinary bladder for diagnostics, which has led to the first application of an in vivo Raman cystoscopy in the past few years. Table 7 shows the Raman capturing methods, laser details, substrates used (where applicable) and the wavenumber range recorded in publications that have used Raman spectroscopy with a fiber-optic probe to diagnose and grade bladder cancer tissue.

In 2005, Crow et al. [46] were the first research group to integrate a fiber-optic probe into Raman spectroscopy to differentiate between benign and malignant bladder tissue in vitro using a diagnostic algorithm. Bladder tissue samples were obtained from 24 patients during TURBT and TURP, and classified as normal, cystitis or TCC by a uropathologist. Raman spectra were obtained by holding the probe in contact with these tissue samples, with an acquisition time of $10 \mathrm{~s}$. Spectra were baseline-corrected and analyzed with PCA and LDA to result in a sensitivity of $89 \%$ and specificity of $79 \%$ for benign tissues, and a sensitivity of $79 \%$ and specificity of $89 \%$ for malignant tissues, thus showing the suitability of using a fiber-optic probe for the diagnosis of bladder cancer. The probe used in this study was designed to be compatible with the working channel of a standard rigid or flexible cystoscope so that it could be easily integrated into in vivo studies, such as during endoscopic, laparoscopic or open procedures. While it is proposed that the probe could be used in vivo, the reported experimental work focused on bladder tissue in vitro as a proof of concept.

In 2009, Grimbergen and colleagues [47] also investigated the potential of using Raman spectroscopy for bladder tissue diagnosis during cystoscopy by examining bladder tissue biopsies ex vivo using an endoscopic probe. In total, 107 bladder tissue samples were obtained from 54 patients during TURBT, where 5-ALA was used to enhance tissue contrast. Samples were graded as normal, cystitis,
CIS, G1 TCC, G2 TCC, G3 TCC and atypia by a pathologist. Raman spectral measurements were obtained from fresh tissue samples (immediately after surgery) with an integration time of $2 \mathrm{~s}$. Spectra were pre-processed and a model was developed to distinguish between normal and malignant tissue using PCA and LDA techniques with a leave-one-out cross validation method resulting in a sensitivity and specificity of $78.5 \%$ and $78.9 \%$ respectively. A seven-group algorithm to distinguish between each type of pathology was also investigated, but this appeared to show a greater number of misclassifications. Whilst this study was based on ex vivo experimentation, these results show the possibility of using a Raman spectroscopic probe for discerning normal from malignant bladder tissue in vivo in the presence of 5-ALA.

Following from the two previous studies [46, 47] involving the application of Raman fiber-optic probes to in vitro and ex vivo bladder tissue, in 2010, Draga et al. [8] were the first research group to investigate the use of a Raman-based fiber-optic probe for the diagnosis of bladder cancer in vivo. Raman spectra were obtained during TURBT procedures on 17 patients without any photosensitive dyes, 12 patients with 5-ALA and 9 patients with HAL, representing a mixture of normal, Ta, T1 and T2 bladder tissues - the pathologic staging was later confirmed by two pathologists from biopsy samples taken from each patient during the procedure. Spectra were measured with a high-volume Raman probe with a penetration depth of $2 \mathrm{~mm}$ for integration times of $1-5 \mathrm{~s}$. All spectra were baseline-corrected and normalized, and three diagnostic algorithms were designed using PCA, LDA and a leave-one-out cross validation method. The first algorithm was able to distinguish between normal and cancerous tissue; the second between normal, non-invasive TCC (Ta) and invasive TCC (T1/T2); and the third between normal, inflammation and cancer. The first algorithm resulted in a sensitivity of $85 \%$ and a specificity of $79 \%$, and the results of the other two algorithms can be found in Table 8. It is of note that the authors have suggested that the lower sensitivities and specificities might be due

Table 7 Capture method, substrates and wavelengths used for Raman spectroscopy-based diagnostic model for diseases associated with bladder tissue.

\begin{tabular}{lllrrrr}
\hline References & Year & $\begin{array}{l}\text { Raman optical } \\
\text { method }\end{array}$ & $\begin{array}{r}\text { Laser } \\
\text { wavelength (nm) }\end{array}$ & $\begin{array}{r}\text { Laser power [at } \\
\text { sample] (mW) }\end{array}$ & $\begin{array}{l}\text { Substrate (if } \\
\text { applicable) }\end{array}$ & $\begin{array}{c}\text { Wavenumber } \\
\left.\text { range (cm }{ }^{-1}\right)\end{array}$ \\
\hline$[46]$ & 2005 & Fiber-optic (in vitro) & 785 & {$[70]$} & - & $800-1800$ \\
{$[47]$} & 2009 & Fiber-optic (ex vivo) & 785 & NS & Aluminum foil & $400-1800$ \\
{$[8]$} & 2010 & Fiber-optic (in vivo) & 785 & {$[56-89]$} & - & $400-1800$ \\
{$[9]$} & 2012 & Fiber-optic (ex vivo) & 785 & {$[30]$} & - & $400-1700$ \\
\hline
\end{tabular}

NS, not specified. 
Table 8 Results achieved in algorithms designed by Draga et al. [8] for in vivo diagnosis of different bladder pathologies.

\begin{tabular}{lcr}
\hline Pathology & Sensitivity (\%) & \multicolumn{2}{c}{ Specificity (\%) } \\
\hline (i) Three-group algorithm: & & 85 \\
$\quad$ Normal & 79 & 83 \\
$\quad$ Non-invasive TCC & 41 & 76 \\
$\quad$ Invasive TCC & 58 & 89 \\
(ii) Three-group algorithm: & & 71 \\
$\quad$ Normal & 78 & 87 \\
$\quad$ Inflammation & 60 & \\
Cancer & 71 & \\
\hline
\end{tabular}

TCC, transitional cell carcinoma.

to an insufficient sample size. The presence of a photosensitive dye (5-ALA or HAL) was discriminated from tissue without any dye with a sensitivity and specificity of $85 \%$ and 69\%, respectively. Diagnosis of normal and cancerous bladder tissue in the absence of 5-ALA and HAL resulted in a sensitivity of $88 \%$ and specificity of $79 \%$, compared to a sensitivity of $78 \%$ and specificity of $70 \%$ in the presence of 5-ALA or HAL. Increasing the acquisition time was also evaluated, but it was found that longer acquisition times did not considerably change the overall predication accuracy ( $t \leq 3 \mathrm{~s}$ : sensitivity: $85 \%$, specificity: $79 \%$; $t \geq 3 \mathrm{~s}$ : sensitivity: $86 \%$, specificity: $78 \%$ ). Here, Draga et al. [8] have successfully demonstrated the use of Raman spectroscopy for the diagnosis of bladder cancer in vivo, both with and without the presence of a photosensitive dye (5-ALA and HAL). This shows the potential of integrating this Raman probe into fluorescence cystoscopy procedures to improve diagnosis, and further improvements into the design of the fiber-optic probe might increase the overall diagnostic accuracy in order to achieve results comparable to those found in previous in vitro studies.

And most recently, in 2012, Barman et al. [9] proposed the use of a confocal fiber-optic Raman probe to increase the specificity (in terms of tissue depth discrimination) for bladder cancer diagnosis in this proof-of-concept study performed ex vivo. Bladder tissue samples were excised from 14 patients during TURBT, and confirmed as being normal or cancerous by a senior uropathologist. Raman spectra were obtained from each sample (ex vivo) straight after surgery using both a high-volume probe and a confocal probe to compare the sensitivities and specificities achieved for each. The confocal probe was designed by placing a pinhole aperture into the high-volume probe to decrease the depth of field from $520 \mu \mathrm{m}$ to $280 \mu \mathrm{m}$, thus suppressing the spectral information from surrounding regions and from deeper tissue layers beyond the region of interest. All spectra were pre-processed and diagnostic algorithms were developed using PCA and logistic regression analysis along with a leave-one-out cross validation. The high-volume probe produced a sensitivity of $85.7 \%$ and specificity of $85.7 \%$, whereas the confocal probe has a sensitivity of $85.7 \%$ and specificity of $100 \%$. It should be noted that this diagnostic algorithm was based on only two PCs, thus emphasizing the robustness of the confocal Raman probe. The significant increase in specificity values of the confocal probe in comparison to the high-volume probe are associated with the smaller depth of field values, giving this particular device an advantage in the application of Raman probes for real-time in vivo diagnosis of bladder pathology. This confocal probe has also shown potential to be used to discriminate between different grades and stages of tumors that might improve the overall pathological diagnosis.

In summary, in this section it has been shown that Raman spectroscopy can be used to accurately discriminate between healthy and cancerous bladder tissue, as well as being able to classify the exact grade (or stage) of the tissue sample, both in vivo and in vitro. This development paves the way for the potential integration of Raman spectroscopy into a clinical setting to improve upon the accuracy of diagnosing bladder cancer, and therefore, have the ability to improve upon patient outcome. Another advantage of this technique is that it is minimally invasive, and can applied in vivo during a cystoscopic procedure or in vitro from biopsy samples as discussed in the previous section. Table 9 provides a summary of all results to date generated using Raman spectroscopic techniques for bladder tissue diagnosis applied in vivo and in vitro/ex vivo. These results clearly show the potential of this robust technique becoming a standard clinical procedure for highly accurate bladder disease diagnosis.

\subsection{Raman cytology}

Within the last 5 years, studies have begun investigating the potential use of Raman spectroscopy for analyzing and classifying disease from bladder cells rather than tissue, thus moving to a completely non-invasive procedure of detecting bladder disease from voided urine. This technique has the potential of replacing standard biopsy or cytology procedures. In this section the current progress in this area is reviewed and the results achieved using different Raman optical methods are highlighted.

To prepare bladder cells for Raman spectral measurements, cells were either cultured from cell lines (for proof of concept studies) or obtained from voided urine. Cell lines are cultured within a nutrient medium (typically containing fetal calf serum). These cells are then removed, 
Table 9 List of tissue details, statistical methods used and results achieved from each referenced study in the development of a diagnostic model for bladder cancer in tissue, either using Raman microspectroscopy or a Raman fiber-optic probe.

\begin{tabular}{|c|c|c|c|c|c|c|c|}
\hline References & Source & Storage & $\begin{array}{r}\text { Number of } \\
\text { patients } \\
\text { (samples) }\end{array}$ & Tissue status & Statistics & Sensitivity (\%) & Specificity (\%) \\
\hline [8] & TURBT & $\begin{array}{l}\text { In vivo } \\
\text { With 5-ALA } \\
\text { With HAL } \\
\text { Without PDD }\end{array}$ & $\begin{array}{r}12 \\
9 \\
17\end{array}$ & $\begin{array}{l}\text { Mixture of normal, } \\
\text { Ta, T1 and T2 }\end{array}$ & PCA, LDA & 85 & 79 \\
\hline [9] & TURBT & Fresh samples & $\begin{array}{r}14(14) \\
(14)\end{array}$ & $\begin{array}{l}\text { Normal } \\
\text { Cancerous }\end{array}$ & PCA, LRA & 85.7 & 100.0 \\
\hline [11] & Biopsy & $\begin{array}{l}\text { Without 5-ALA } \\
\text { Snap-frozen to }-80^{\circ} \mathrm{C}\end{array}$ & $\begin{array}{r}73(26) \\
(14) \\
(2) \\
(14) \\
(25) \\
(11)\end{array}$ & $\begin{array}{l}\text { Normal } \\
\text { Cystitis } \\
\text { CIS } \\
\text { G1 } \\
\text { G2 } \\
\text { G3 }\end{array}$ & PCA, LDA & 88.0 & 80.1 \\
\hline [11] & Biopsy & $\begin{array}{l}\text { With 5-ALA } \\
\text { Snap-frozen to }-80^{\circ} \mathrm{C}\end{array}$ & $\begin{array}{r}19(20) \\
(6) \\
(1) \\
(3) \\
(6) \\
(2)\end{array}$ & $\begin{array}{l}\text { Normal } \\
\text { Benign } \\
\text { CIS } \\
\text { G1 } \\
\text { G2 } \\
\text { G3 }\end{array}$ & PCA, LDA & $\begin{array}{r}75.4 \\
100.0(\mathrm{FL}+\text { Raman })\end{array}$ & $\begin{array}{r}88.7 \\
80.8 \text { (FL+Raman) }\end{array}$ \\
\hline [43] & Cystoscopy & Snap-frozen to $-80^{\circ} \mathrm{C}$ & $12(12)$ & $\begin{array}{l}\text { Normal } \\
\text { CIS } \\
\text { LGC } \\
\text { MGC } \\
\text { HGC }\end{array}$ & PCA, LDA & $\begin{array}{l}93.0 \\
83.0 \\
96.0 \\
78.0 \\
98.0\end{array}$ & $\begin{array}{l}99.0 \\
99.0 \\
96.0 \\
98.0 \\
96.0\end{array}$ \\
\hline [44] & $\begin{array}{l}\text { TURBT, TURP and } \\
\text { cystoscopy }\end{array}$ & Snap-frozen to $-80^{\circ} \mathrm{C}$ & $\begin{array}{r}22(22) \\
10(10) \\
2(2) \\
9(9) \\
20(21) \\
7(9) \\
1(1) \\
1(1)\end{array}$ & $\begin{array}{l}\text { Normal } \\
\text { Cystitis } \\
\text { CIS } \\
\text { G1 } \\
\text { G2 } \\
\text { G3 } \\
\text { G3 squamous } \\
\text { G3 adenocarcinoma }\end{array}$ & PCA, LDA & $\begin{array}{r}95.0 \\
87.0 \\
84.0 \\
71.0 \\
78.0 \\
92.0 \\
100.0 \\
92.0\end{array}$ & $\begin{array}{r}98.0 \\
98.0 \\
99.0 \\
95.0 \\
94.0 \\
98.0 \\
99.0 \\
100.0\end{array}$ \\
\hline [45] & NS & $\begin{array}{l}\text { Snap-frozen } \\
\text { (temperature NS) }\end{array}$ & $\begin{array}{l}2(2) \\
9(9) \\
1(1) \\
3(3)\end{array}$ & $\begin{array}{l}\text { Normal } \\
\text { T2-T3 } \\
\text { TIS } \\
\text { Cystitis }\end{array}$ & $\begin{array}{l}\text { PCA, LDA, } \\
\text { CAS, HCA }\end{array}$ & 94.0 & 92.0 \\
\hline [46] & $\begin{array}{l}\text { Cystoscopy, } \\
\text { TURBT and TURP }\end{array}$ & Snap-frozen to $-80^{\circ} \mathrm{C}$ & $\begin{array}{r}24(11) \\
(8) \\
(2) \\
(7) \\
(1)\end{array}$ & $\begin{array}{l}\text { Normal } \\
\text { Cystitis } \\
\text { G1 } \\
\text { G2 } \\
\text { G3 }\end{array}$ & PCA, LDA & $\begin{array}{r}89.0 \text { (benign) } \\
79.0 \text { (malignant) }\end{array}$ & $\begin{array}{r}79.0 \text { (benign) } \\
89.0 \text { (malignant) }\end{array}$ \\
\hline [47] & TURBT & Fresh samples & $\begin{array}{r}54(42) \\
(16) \\
(14) \\
(3) \\
(15) \\
(10) \\
(7)\end{array}$ & $\begin{array}{l}\text { Normal } \\
\text { Cystitis } \\
\text { CIS } \\
\text { G1 } \\
\text { G2 } \\
\text { G3 } \\
\text { Atypia }\end{array}$ & PCA, LDA & 78.5 & 78.9 \\
\hline
\end{tabular}

TURBT, transurethral resection of bladder tumor; TURP, transurethral resection of the prostate; FL, fluorescence cystoscopy; 5-ALA, 5-aminolevulinic acid; HAL, hexaminolevulinate; PDD, photodynamic diagnosis; PCA, principal components analysis; LDA, linear discriminant analysis; LRA, logistic regression analysis; CAS, cluster averaged spectra; HCA, hierarchical cluster analysis.

Ta, T1, T2, and T3, tumor states according to Table 2; TIS/CIS, carcinoma in situ; G1, low grade; G2, medium grade; G3, high grade; LGC, low-grade carcinoma; MGC, medium-grade carcinoma; HGC, high-grade carcinoma.

NS, not specified. 
Table 10 Capture method, substrates and wavelengths used for Raman spectroscopy in analyzing bladder cells.

\begin{tabular}{lllrrrr}
\hline References & Year & $\begin{array}{l}\text { Raman optical } \\
\text { method }\end{array}$ & $\begin{array}{r}\text { Laser } \\
\text { wavelength }(\mathbf{n m})\end{array}$ & $\begin{array}{r}\text { Laser power [at } \\
\text { sample] (mW) }\end{array}$ & $\begin{array}{r}\text { Substrate (if } \\
\text { applicable) }\end{array}$ & $\begin{array}{r}\text { Wavenumber } \\
\left.\text { range (cm }{ }^{-1}\right)\end{array}$ \\
\hline$[36]$ & 2008 & Tweezers & 514.5 & $\mathrm{NS}$ & - & $\mathrm{NS}$ \\
{$[32]$} & 2009 & Tweezers & 514.5 & $400[100], 200[32.6]$ & - & $\mathrm{NS}$ \\
{$[12]$} & 2011 & Conventional & 532 & $\mathrm{NS}$ & Aluminum & $350-3560^{\text {a }}$ \\
{$[13]$} & 2011 & Modulated & 785 & 100 [8] & Quartz & $500-2100$ \\
\hline
\end{tabular}

${ }^{a}$ Exact wavenumber range not given, assumption made from Raman spectra provided.

NS, not specified.

and can be analyzed live or after a chemical fixation has been applied. It is also possible to suspend cells in a urine solution in order to observe the effects of urine on these cells. Otherwise, bladder cells can be acquired from fresh voided urine via a centrifugation process.

To analyze bladder cells using Raman spectroscopy, the cells can be deposited onto a substrate using a cytospin or they can be analyzed using an optical Raman tweezers technique which isolates a cell within an optical trap, and allows for Raman spectral data only to be obtained from the individual cell, thus reducing background contributions. Table 10 displays the Raman optical methods applied, the details of the laser used, substrates used (if any) and the wavenumber range over which data were collected and analyzed in studies investigating the use of Raman spectroscopic methods to diagnose bladder cancer from urothelial cells.

The first study to analyze bladder cells using Raman spectroscopy was investigated by Harvey et al. [36] in 2008, where Raman optical tweezers were used to trap and analyze both live and chemically fixed bladder cells, and to differentiate between normal and cancerous cells. In the first part of this study, the Raman tweezers technique was applied to discriminate between a malignant bladder cell line (MGH-U1 - otherwise known as T24 [48]) and a malignant prostate cell line (PC-3). All of the cells were fresh and stored in phosphate buffered saline (PBS) before and during measurement. Raman spectra were obtained using a $514.5 \mathrm{~nm}$ laser, and all spectra were pre-processed by removing the background contribution, followed by extended multiplicative signal correction. PCA was applied to the spectra and the resulting PCA plot of this shows that the two cell types can be identified as separate cell groups. The second part of the study investigated the use of Raman tweezers to discriminate between the following alcohol-fixed cell lines: malignant bladder cells (MGH-U1), malignant prostate cells (LNCaP, PC-3) and benign prostate cells (BPH). All cells fixed using SurePath ${ }^{\mathrm{TM}}$ (BD Diagnostics, NJ, USA) and stored at $<4^{\circ} \mathrm{C}$. Raman spectra were recorded and pre-processed the same way as for live cells. Spectra were then subjected to PCA and LDA to differentiate between each cell group based on a training and test validation method, the results of which are shown in Table 11. This initial study based on the application of Raman tweezers to differentiate between different urological cell lines has shown the diagnostic potential of this technique for detecting both bladder and prostate cancer.

In 2009, Harvey et al. [32] continued on from this initial study to further investigate the application of Raman tweezers to classify cultured cell lines that have been chemically fixed using either SurePath ${ }^{\mathrm{TM}}$ or formalin, as well as investigating the effect of exposure to urine on these cells. Raman tweezers were first used to distinguish between the following cell lines fixed with SurePath $^{\mathrm{TM}}$ : malignant bladder cells (MGH-U1), malignant prostate cells (LNCaP, PC-3), normal prostate cells (PNT2$\mathrm{C} 2$ ) and benign prostate cells (BPH). The Raman tweezers configuration consisted of two lasers, whereby cells were trapped and analyzed using a $514.5 \mathrm{~nm}$ laser (laser power of $200 \mathrm{~mW}$ used; $32.6 \mathrm{~mW}$ at sample surface), and a secondary $1064 \mathrm{~nm}$ laser was used to retain the cell in the optical trap. Raman spectra were obtained in this method from 100 MGH-U1, 104 BPH, 104 LNCaP, 115 PC-3 and 110 PNT2-C2 cells. All spectra were pre-processed by removing the background contribution, adding a correction factor to adjust the $880 \mathrm{~cm}^{-1}$ Raman peak to the baseline (the author suggests that this peak is most likely contamination from

Table 11 Results achieved by Harvey et al. [36] using Raman tweezers to discriminate between fixed cell lines using PCA and LDA.

\begin{tabular}{lrr}
\hline Cell line & Sensitivity (\%) & Specificity (\%) \\
\hline Bladder cancer (MGH-U1) & 91.7 & 96.4 \\
Benign prostate (BPH) & 93.8 & 97.1 \\
Prostate cancer (PC-3) & 72.7 & 98.2 \\
Prostate cancer (LNCaP) & 90.2 & 94.5 \\
\hline
\end{tabular}


the SurePath ${ }^{\mathrm{TM}}$ solution). Extended multiplicative scattering correction, vector normalization and mean centering were also applied. A diagnostic model was designed by applying PCA and LDA to the spectra, and the results can be seen in Table 12, showing that is it possible to clearly distinguish between each group of cells after being fixed with SurePath ${ }^{\mathrm{TM}}$. However, the authors have noted that the MGH-U1 and PC-3 cells were found to be larger in SurePath $^{\mathrm{TM}}$ than when unfixed.

The second section of this study was based on the use of Raman tweezers to distinguish between the following formalin-fixed cells: malignant bladder cells (MGH-U1), urethral cells (from patients), benign prostate cells (BPH), normal prostate cells (PNT2-C2) and malignant prostate cells (LNCaP, PC-3). For these experimental measurements, the laser power was increased to $400 \mathrm{~mW}$ (100 $\mathrm{mW}$ at the sample surface), and as a result, a secondary, counter-propagating beam was not required for these cells. Raman spectra were recorded from 135 cells in each group, and spectra were pre-processed using a 1:1 background subtraction, extended multiplicative scattering correction, vector normalization and mean centering. For classification, PCA and LDA were applied, with blind and double blind testing, to yield the results shown in Table 12.

Further classification models (based on classifying four cells at a time) were also developed, the results of which can be found elsewhere [32] also show good separation between each cell type. A comparison between both chemical fixing methods shows that formalin has a better diagnostic performance and a greater $\mathrm{S} / \mathrm{N}$, although this is

Table 12 Results achieved by Harvey et al. [32] using Raman tweezers to discriminate between different cell lines that have been fixed with SurePath ${ }^{\mathrm{TM}}$ or formalin.

\begin{tabular}{lrr}
\hline Cell line & Sensitivity (\%) & Specificity (\%) \\
\hline SurePath'M fixed: & & \\
MGH-U1 & 93.8 & 92.1 \\
BPH & 79.2 & 99.1 \\
PNT2-C2 & 72.7 & 94.5 \\
PC-3 & 95.1 & 95.7 \\
LNCaP & 78.8 & 97.8 \\
Formalin fixed: & & \\
MGH-U1 & 88.2 & 98.1 \\
Urethral cells & 97.8 & 98.1 \\
BPH & 100.0 & 99.2 \\
PNT2-C2 & 99.5 & 98.4 \\
PC-3 & 98.0 & 99.6 \\
LNCaP & 93.5 & 99.6 \\
\hline
\end{tabular}

MGH-U1, malignant bladder cells; BPH, benign prostate cells; PNT2C2, normal prostate cells; PC-3 and LNCaP, malignant prostate cells. due to the higher laser power used. There was also no contamination in spectra from the formalin solution, whereas a significant contribution was found in the SurePath ${ }^{\mathrm{TM}}$ spectra.

Finally, the last part of this study was to determine the effects of exposure to urine on the ability to classify cells. To investigate this, all cells (MGH-U1, BPH, LNCaP and PC-3) were unfixed and stored at room temperature before being exposed to urine for the following time durations: $15 \mathrm{~min}, 30 \mathrm{~min}, 45 \mathrm{~min}, 1 \mathrm{~h}, 2 \mathrm{~h}, 4 \mathrm{~h}, 6 \mathrm{~h}, 8 \mathrm{~h}, 10 \mathrm{~h}$ and $12 \mathrm{~h}$. Raman spectra were obtained using the same set-up as used for the formalin-fixed cells, and spectra were also pre-processed using the same method. PCA and LDA were applied to the spectra, and it was shown that in general the prediction values do not deteriorate over $12 \mathrm{~h}$. On the contrary, a drop in prediction values was noted between $30 \mathrm{~min}$ and 45-60 min, the author contributed this to a reduction in the stress-response proteins; otherwise results were similar to before urine exposure. Overall, this study has shown the potential of using Raman tweezers to distinguish between bladder cells and prostate cells, and also between cancerous and non-cancerous cells. It has also been shown that it is possible to accurately classify cells that have been fixed with SurePath $^{\mathrm{TM}}$ and formalin, and that up to $12 \mathrm{~h}$ exposure to urine does not affect the ability to distinguish between the different cell groups.

In 2011, Shapiro et al. [12] investigated the possibility of applying Raman spectroscopy to bladder epithelial cells from voided urine in order to determine whether or not TCC was present. For this study, fresh urine samples were obtained from 340 patients (116 without TCC, 92 with low-grade TCC and 132 with high-grade TCC). To prepare the urine samples for Raman spectroscopic measurements, they were spun at $3000 \mathrm{rpm}$ for $5 \mathrm{~min}$, the supernatant was removed, and the pellet was resuspended in distilled water. It was then centrifugated at $3000 \mathrm{rpm}$ for $5 \mathrm{~min}$ again and the remaining supernatant removed. Filtration was carried out to capture the bladder epithelial cells and to wash off other constituents. The epithelial cells were washed to resuspend them before being placed into a cytospin chamber and spun at $1100 \mathrm{rpm}$ for $5 \mathrm{~min}$ and deposited onto an aluminum slide. Raman spectra were obtained from an average of five cells per slide using a $532 \mathrm{~nm}$ laser; all spectra were baseline-corrected and normalized. The resulting spectra showed a positive $1584 \mathrm{~cm}^{-1}$ Raman peak for cancerous cells that was not present for non-malignant cells. The authors developed a model based only on this peak (no PCA or LDA required) that could classify spectra as normal, low-grade TCC or high-grade TCC by evaluating 
the height of the $1584 \mathrm{~cm}^{-1}$ peak above the baseline at $1500 \mathrm{~cm}^{-1}$ and using a set of specific thresholds to classify each spectrum with an overall sensitivity of $92 \%$ and a specificity of $91 \%$. A second part of this study involved comparing the Raman spectra from these bladder cells with spectra obtained from bladder tissue samples. These bladder tissue samples were obtained from surgical touch preps and were frozen prior to Raman measurements. This study showed that the spectra from bladder cells and tissue were virtually identical, and that it is possible to determine the histological grade of both cells and tissue based on the height of the distinct Raman peak at $1584 \mathrm{~cm}^{-1}$.

In 2011, Canetta et al. [13] developed the first application of modulated Raman spectroscopy (MRS) for the identification of human bladder cell lines in urine, whilst monitoring cell viability after exposure to urine, and the differences between standard Raman spectroscopy (SRS) and MRS for cell classification were also examined. Healthy human urothelial cells (SV-HUC-1) and malignant bladder cells (MGH-U1) were cultured, and then exposed to a solution containing two parts fresh urine and one part PreservCyt (Cytec, UK) for the following time durations: $0 \mathrm{~h}$ (control), $30 \mathrm{~min}, 3 \mathrm{~h}$ and $6 \mathrm{~h}$. This was then centrifugated for $10 \mathrm{~min}$ and the cells were resuspended in PreservCyt for storage. Prior to Raman spectral measurements, the cells were washed with PBS before being resuspended in $100 \mu \mathrm{l}$ of PBS and placed in a sample chamber between a quartz slide and a quartz coverslip. Cells were allowed $\sim 30$ min to sediment onto the quartz before Raman measurements were obtained. Spectra were recorded from 40 urothelial cells and 40 bladder cancer cells for each of the urine exposure times using a $785 \mathrm{~nm}$ modulated laser (modulated with a ramp wave of $50 \%$ symmetry, $40 \mathrm{mHz}$ modulation frequency, $60 \mathrm{GHz}$ modulation amplitude - corresponding to $0.2 \mathrm{~nm}$ ). For each cell, 40 stacked spectra were recorded with a total integration time of $200 \mathrm{~s}$. PCA was applied to the spectra and a non-specified algorithm was used for classification, the results of which can be seen in Table 13. These results show that exposure to urine affects the cell's biochemistry causing a reduction in the $\mathrm{S} / \mathrm{N}$ in the spectra. However, it is still possible to distinguish between both cell groups using MRS, even after exposure to urine for $6 \mathrm{~h}$.

The second part of this study involved monitoring cell viability in urine using 1\% Fast Green dye (Sigma Chemical Company, MO, USA), and it was found that after $1 \mathrm{~h}$, the viability of the cancer cells decreased to $\sim 60 \%$ and $\sim 64 \%$ for healthy cells, and the reproductive capacity of both cells decreased rapidly after exposure
Table 13 Results obtained from Canetta et al. [13] for classifying fixed urothelial cells (SV-HUC-1) and fixed bladder cancer cells (MGH-U1), for assigned exposure to urine times, using both standard Raman spectroscopy and modulated Raman spectroscopy.

\begin{tabular}{lrrr}
\hline Method & $\begin{array}{r}\text { Urine exposure } \\
\text { times (h) }\end{array}$ & $\begin{array}{r}\text { Sensitivity } \\
\text { (\%) }\end{array}$ & $\begin{array}{r}\text { Specificity } \\
\text { (\%) }\end{array}$ \\
\hline $\begin{array}{l}\text { Modulated Raman } \\
\text { spectroscopy (PCA) }\end{array}$ & 0 & 98 & 95 \\
$\begin{array}{l}\text { Modulated Raman } \\
\text { spectroscopy (PCA) }\end{array}$ & 6 & 80 & 87 \\
$\begin{array}{l}\text { Standard Raman } \\
\text { spectroscopy (PCA } \\
\text { and Fourier Transform) }\end{array}$ & 0 & 97 & 72 \\
\hline
\end{tabular}

PCA, principal components analysis.

to urine. This mirrors the Raman data obtained from different exposure times where changes in the biochemistry can be seen with progressing exposure times. The robustness of MRS in comparison to SRS was then investigated by comparing the Raman spectra obtained from each method for fixed urothelial cells and fixed bladder cancer cells not exposed to urine (control group). The SRS spectra showed a strong autofluorescence signal from the quartz substrate, and Raman peaks from the cells are not well defined. On the other hand, the MRS spectra allow for Raman peaks to be clearly observed. PCA was applied to both the SRS and MRS spectra to discriminate between both cell groups, and the Fourier Transform was also applied to the SRS spectra in order to distinguish between the slow varying background and the Raman peaks, the results of which can also be seen in Table 13. In the Fourier domain, the background signals were present at low frequencies, whereas the Raman signals were present at high frequencies; therefore the Fourier Transform was able to separate the most important Raman signals from the background. Higher sensitivities and specificities were achieved with MRS, which may be due to the better $\mathrm{S} / \mathrm{N}$ obtained by filtering out the Raman peaks associated with the biological cells from the fluorescence background signals. It has also been noted that the healthy urothelial cells show relatively strong protein and carbohydrate Raman peaks (1100-1300 $\mathrm{cm}^{-1}$ region) in comparison to the cancerous cells, and there was an increase in ring breathing modes for the cancerous cell spectra $(669,727,785,828,1095$, $1578 \mathrm{~cm}^{-1}$ ) indicating a higher concentration of deoxyribonucleic acid (DNA) in malignant cells.

In 2013, Praveen et al. [49] further developed modulated Raman spectroscopy for the analysis of bladder cancer cells, with the aim of reducing acquisition 
times in order to achieve high throughput cell screening. Healthy human urothelial cells (SV-HUC-1) and malignant bladder cells (MGH-U1) were cultured, and placed into a sample chamber built from a quartz slide and a quartz cover slip. In this study, various experimental parameters were varied to minimize acquisition time whilst maintaining complete discrimination between both cell groups (using PCA). The parameters under investigation were the range within which the wavelength was modulated, number of cycles, sampling rate and acquisition time. Results from this study have shown that the optimum modulation amplitude was $\Delta \lambda=0.32 \mathrm{~nm}(\Delta v=160 \mathrm{GHz})$ or greater, and that the sample rate did not affect the $\mathrm{S} / \mathrm{N}$ of the Raman peaks provided there were three or more wavelengths being sampled. Using these parameters, the authors were able to discriminate between healthy and cancerous bladder cells with a total acquisition time of $6 \mathrm{~s}$ (i.e. $2 \mathrm{~s}$ per acquisition cycle at a sampling rate of 3), with a laser power of $200 \mathrm{~mW}$ maintained at the sample. This study has shown the potential of using modulated Raman spectroscopy for the classification of bladder cells with a significantly shorter acquisition time, thus highlighting the potential use of this technique for higher throughput Raman measurements based on flow cytometry.

In summary, it has been shown that Raman optical tweezers can be used to discriminate between normal and cancer bladder cell lines, and also between bladder and prostate cell lines. This method has been applied to live cells, cells chemically fixed (with formalin or SurePath ${ }^{\mathrm{TM}}$ ), and cells that have been exposed to urine for up to $12 \mathrm{~h}$, all resulting in high classification prediction values. Conventional Raman microspectroscopy has also been applied to discriminate between normal and cancerous cells obtained from voided urine based on thresholding the Raman peak at $1584 \mathrm{~cm}^{-1}$, which is seen in both the spectra from bladder cells and bladder tissue samples. In study by Canetta et al. [13] (the only one to date using cells from voided urine) Raman microspectroscopy was shown to have diagnostic sensitivity of $92 \%$ and specificity of $91 \%$. And finally, it is possible to differentiate between normal and cancerous cell lines using SRS and MRS, when cells have been fixed or incubated in urine for up to $6 \mathrm{~h}$, the latter showing improved results for using relatively inexpensive quartz substrates. It has been noted that while urine affects the cells biochemistry, the results obtained are similar to those of control cells [12, 32]. Thus, this shows how robust Raman spectroscopic techniques are for discriminating between bladder cells regardless of how they are prepared or stored.

\section{Investigating the etiology of bladder cancer using Raman spectroscopy}

In the last two decades, there has been considerable interest in the use of Raman spectroscopy in identifying the biochemistry of bladder tissue, and the potential of using this to detect differences between the biochemistry of normal tissue with cancerous tissue, which would not only assist in early detection of disease, but also aid in understanding the etiology of bladder cancer. The main methods used for this are the creation of a reference database of component spectra, least squares fitting and Raman spectroscopic mapping. These techniques can be used to highlight regions of different tissue composition, and could be integrated into surgical procedures to identify border regions between normal and diseased tissue sections, thus improving patient outcome. Another application is to identify the biomolecular changes occurring with disease progression. A brief introduction into each of these methods is given below while Table 14 shows the Raman capturing methods, laser details, the substrates used and the wavenumber range recorded in publications that have used Raman spectroscopy to analyze the biochemistry of bladder tissue. Further details from each publication can be found below and a summary of the main Raman shift peaks associated with each pathology is given in Table 15. In the discussion that follows, all tissue samples are human unless otherwise specified.

- Reference database: To create a reference database, spectra are recorded from a selection of Raman active biological compounds (e.g. collagen, DNA, actin, cholesterol). These spectra are stored in a database, and are compared to the spectra obtained from the tissue sample using multivariate techniques or least squares (see below). This method can be used to determine which components are present and absent and in what concentrations, and therefore can be used to identify the biomolecular composition and pathological status of the sample.

- Least squares: Least squares techniques, such as ordinary least squares (OLS) and non-negative least squares (NNLS) have been used for Raman spectral data in urinary bladder diagnostics. These methods are based on a mathematical technique of finding the best fit for a given set of data by minimizing the sum of the squares of the offset points in the dataset. This approach can be used to fit the mean spectra of the reference components to the mean spectra of the tissue sample of interest. The resulting biochemical 
Table 14 Capture method, substrates and wavelengths used for Raman spectroscopy in analyzing the biochemistry of bladder tissue.

\begin{tabular}{|c|c|c|c|c|c|c|}
\hline References & Year & Raman optical method & $\begin{array}{r}\text { Laser } \\
\text { wavelength }(\mathrm{nm})\end{array}$ & $\begin{array}{r}\text { Laser power [at } \\
\text { sample] (mW) }\end{array}$ & $\begin{array}{r}\text { Substrate (if } \\
\text { applicable) }\end{array}$ & $\begin{array}{r}\text { Wavenumber } \\
\text { range }\left(\mathrm{cm}^{-1}\right)\end{array}$ \\
\hline [50] & 1995 & Conventional & 830 & {$[90-480]$} & Aluminum & $800-1800^{a}$ \\
\hline [51] & 2002 & Mapping & 847 & NS & Calcium fluoride & $400-1800$ \\
\hline [51] & 2002 & Fiber-optic (in vivo) & 830 & [170] & - & $800-1800$ \\
\hline [52] & 2003 & Mapping & 845 & NS & Calcium fluoride & $400-1800$ \\
\hline [53] & 2005 & Kerr-gated & 488 & {$[5]$} & $\begin{array}{r}\text { Quartz \& uric } \\
\text { acid }\end{array}$ & $500-1900^{a}$ \\
\hline [45] & 2006 & Mapping & 845 & 100 & Calcium fluoride & $400-1800$ \\
\hline [54] & 2007 & Conventional & 830 & $300[100]$ & Calcium fluoride & $300-1900^{a}$ \\
\hline [11] & 2009 & Conventional & 830 & $300[100]$ & Calcium fluoride & NS \\
\hline [8] & 2010 & Fiber-optic (in vivo) & 785 & [56-89] & - & $400-1800$ \\
\hline [12] & 2011 & Conventional & 532 & NS & Aluminum & $350-3560^{a}$ \\
\hline
\end{tabular}

aExact wavenumber range not given, assumption made from Raman spectra provided.

NS, not specified.

spectral model can indicate the main biochemical components for that tissue. A disadvantage of least squares is the sensitivity of the fit to any collinearity in the components. Therefore it is often necessary to remove some of the components whose spectra vary together in a co-linear manner.

- Raman spectroscopic mapping: Raman spectroscopic mapping is another method of obtaining information from conventional Raman spectroscopy. This is where a 2-D 'map' of the intensity of Raman peaks are recorded with respect to spatial positioning. This is achieved by scanning the biological sample in a raster-mode, and recording spectra from a series of grid-points using confocal Raman microspectroscopy. It is possible to apply further statistical analysis, such as PCA and cluster analysis, to arrange these spectra into distinct groups representing regions of different biochemical composition (such as collagen, muscle, etc.), which can then be applied to generate a colorcoded 'map' of the tissue area indicating regions of similar composition, thus highlighting regions of damaged or diseased tissue.

In 1995, in what is believed is the first study of the biochemistry of bladder tissue using Raman spectroscopy, Feld et al. [50] investigated the potential of using Raman spectroscopy for providing information about the different molecular composition of normal and cancerous human bladder tissue. Bladder tissue samples were obtained during routine surgical procedures, pathology was confirmed by a board-certified pathologist, and samples were stored at $-80^{\circ} \mathrm{C}$ prior to measurements. Tissue samples were kept moist at all times while the Raman spectra were being obtained. From these spectra, it was found that most spectral differences were associated with an increased content of nucleic acid in bladder carcinoma tissues. All spectra were largely dominated by proteins, but bladder cancer spectra were found to contain more nucleic acids than normal tissue and the lipid content of normal tissue was found to be higher than that of cancerous tissues. This initial study demonstrated the potential of using Raman spectroscopy for determining the differences in biochemical composition between both tissue groups and laid the basic ground work for more research in the area.

In 2002, de Jong and colleagues [51] used a combination of Raman spectroscopic mapping (in vitro) and fiber-optic Raman spectroscopy (in vivo and in vitro) to determine the composition of bladder wall tissue obtained from four guinea pigs with healthy bladders. This study was carried out in two parts with the overall goal of investigating the potential of using Raman spectroscopy to analyze the structural changes in bladder wall tissue that might be associated with bladder function. Firstly, Raman spectroscopic maps were created from the three bladder wall layers (i.e. urothelium, lamina propria and muscle layer) in vitro by collecting 500-700 spectra in a 2-D grid with a collection time for each element of $60 \mathrm{~s}$. All spectra were pre-processed by wavenumber calibration, baseline correction and normalization. A combination of PCA and K-means clustering techniques were applied to generate four clusters, representing the urothelium, lamina propria, muscle layer and an absence of tissue (i.e. holes in tissue or tissue edges). Raman spectroscopic measurements obtained from collagen I, collagen III and actin compounds were used for compositional analysis of the tissue maps designed for each bladder wall layer, and it was found that the muscle layer consisted mainly of actin (and myosin), the lamina propria was composed mainly of 
Table 15 Distinctive Raman peaks reported as being associated with each pathology in the below listed references for bladder tissue.

\begin{tabular}{|c|c|c|}
\hline Raman shift $\left(\mathrm{cm}^{-1}\right)$ & Peak assignment & References \\
\hline \multicolumn{3}{|l|}{ Normal tissue } \\
\hline 875 & Hydroxyproline & {$[8]$} \\
\hline 1080 & C-N stretching of proteins, lipids & [8] \\
\hline 1081 & Fatty acids (urothelium) & {$[51]$} \\
\hline $1220-1300$ & Amide III, collagen & [8] \\
\hline 1261 & Fatty acids (urothelium) & [51] \\
\hline 1289 & Fatty acids (urothelium) & {$[51]$} \\
\hline 1303 & $\mathrm{CH}_{3}, \mathrm{CH}_{2}$, lipids, DNA & [8] \\
\hline 1439 & Fatty acids (urothelium) & {$[51]$} \\
\hline 1446 & $\mathrm{CH}_{2}$ deformation, lipids, proteins & [8] \\
\hline 1657 & Amide I, C $=C$ lipid stretch, phospholipids & [8] \\
\hline 1659 & Fatty acids (urothelium) & [51] \\
\hline \multicolumn{3}{|l|}{ Cancer tissue } \\
\hline 680 & DNA & [8] \\
\hline 789 & DNA & [8] \\
\hline 1003 & Phenylalanine & [8] \\
\hline 1170 & Tyrosine & [8] \\
\hline 1180 & DNA & [8] \\
\hline 1208 & Tryptophan, phenylalanine & {$[8]$} \\
\hline 1370 & DNA, lipids & {$[8]$} \\
\hline 1386 & $\mathrm{CH}_{3}$, lipids & [8] \\
\hline $1540-1580$ & Tryptophan, DNA, phenylalanine & {$[8]$} \\
\hline 1584 & - & {$[12]$} \\
\hline $1600-1620$ & Phenylalanine, tyrosine, tryptophan & [8] \\
\hline \multicolumn{3}{|l|}{ Damaged tissue } \\
\hline 857 & Lactate & {$[52]$} \\
\hline 1005 & Proteins & {$[52]$} \\
\hline 1079 & Proteins & {$[52]$} \\
\hline 1321 & Proteins & [52] \\
\hline 1415 & Lactate & {$[52]$} \\
\hline 1447 & Proteins & {$[52]$} \\
\hline 1455 & Lactate & {$[52]$} \\
\hline 1659 & Proteins & {$[52]$} \\
\hline \multicolumn{3}{|l|}{ Inflammation } \\
\hline 526 & - & {$[8]$} \\
\hline 572 & - & [8] \\
\hline 1485 & DNA & {$[8,55]$} \\
\hline 1569 & - & [8] \\
\hline
\end{tabular}

DNA, deoxyribonucleic acid.

collagen, and the urothelium mostly contained fatty acids (1081, 1261, 1299, 1439 and $1659 \mathrm{~cm}^{-1}$ ), with no collagen at all found in the urothelium. The tissue was then stained with hematoxylin and eosin (H\&E), and a standard digital photograph of the region was taken. Distinct similarities between the bladder wall structure in the Raman map and the photograph are clearly seen in Figure 9.

The second part of this study investigated Raman spectroscopic measurements of bladder wall tissue in vivo using a fiber-optic probe in both full and empty guinea pig bladders. Using the fiber-optic set-up, a reference database of component spectra was initially created using isolated bladder wall layers (urothelium, lamina propria and muscle layer) and guinea pig urine in vitro. This database was then used to determine the relative signal contribution of each tissue layer during in vivo measurements using PCA, K-means clustering and NNLS fitting techniques. For full bladder measurements, $51 \%$ of the Raman spectrum was from urine and following subtraction of this signal, the remaining Raman signal was found to consist of $65 \%$ muscle, 16\% lamina propria and 19\% urothelium. This study of the biochemistry of bladder tissue was the first to employ a compositional analysis of bladder tissue using Raman spectra. The study demonstrated that bladder wall layers are easily distinguishable using Raman spectroscopy techniques, both in vivo and in vitro, even when 

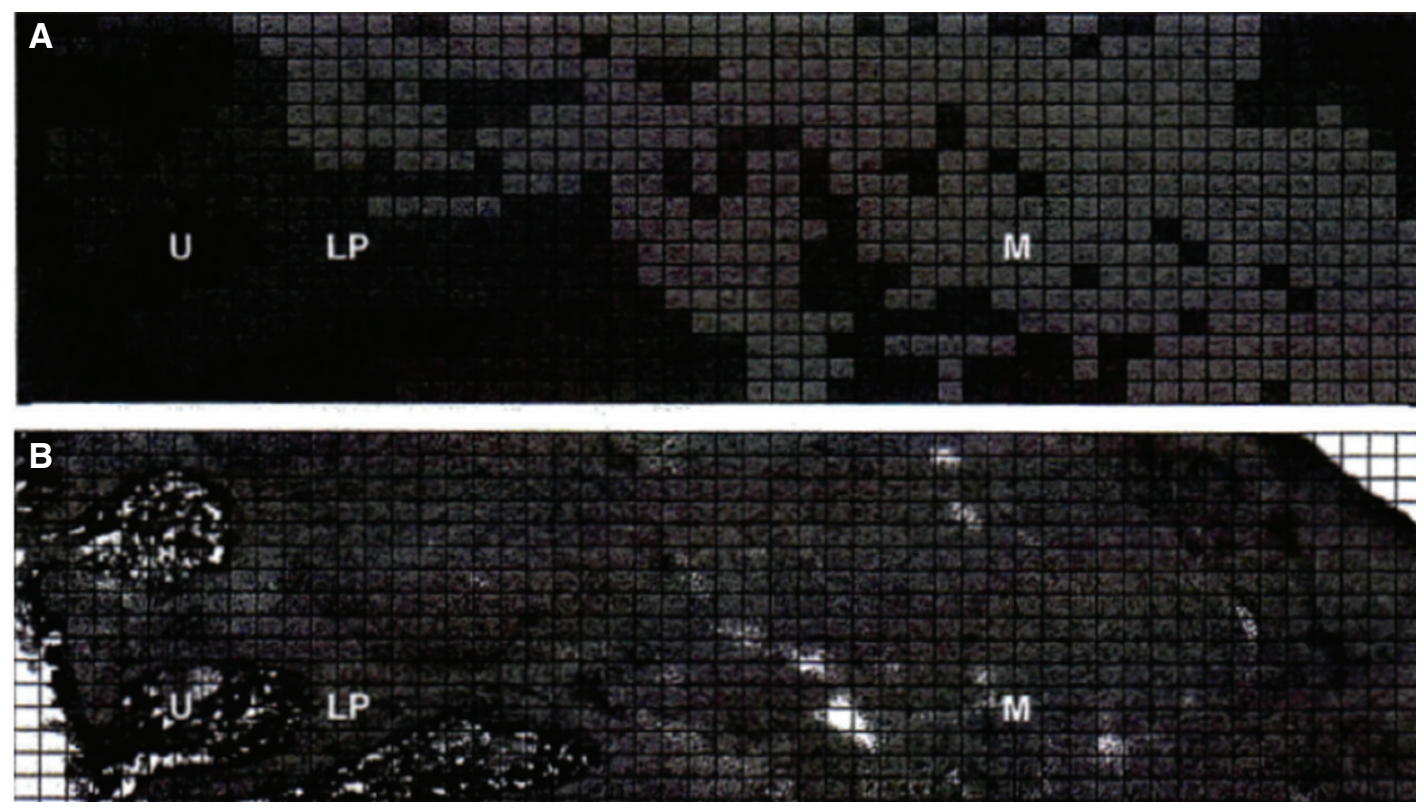

Figure 9 Comparison of bladder wall tissue observed with (A) Raman spectroscopic mapping, and (B) H\&E staining taken with standard photography - indicating regions of urothelium (U), lamina propria (LP) and muscle (M). Images obtained from de Jong et al. [51], reproduced with permission from Elsevier.

urine is present. The results shown in the paper indicated the potential for the use of Raman microspectroscopy, Raman mapping, and Raman-based compositional analysis for monitoring the changes in biomolecular composition of bladder wall tissue and the ability to relate these to changes in bladder functionality.

In 2003, de Jong et al. [52] further investigated the potential of Raman spectroscopic mapping by applying it to unfixed sections of bladder wall tissue, obtained from guinea pigs, in order to determine the differences between normal and damaged bladder wall composition caused by partial urethral obstruction. Raman maps were created from four normal bladders and three obstructed bladders, whereby each grid element spanned $2-3 \mu \mathrm{m}^{2}$ of tissue with a signal collection time of $20 \mathrm{~s}$ that was used to obtain an area averaged spectrum. All spectra were initially baseline-corrected and wavenumber calibrated, and a combination of PCA and K-means clustering were applied to obtain Raman maps that grouped together, and color-coded, the spectral characteristics of different tissue regions in a 2-D grid. As in their previous study, the Raman maps were compared with digital photographs of the same tissue region stained with $\mathrm{H} \& \mathrm{E}$, and the contours of the tissue maps match the image as can be seen in Figure 10. From the compositional analysis, it was found that the healthy tissue map contained only two clusters, one mainly showing characteristics of collagen, the second showing a mixture of actin and myosin. PCA and K-means clustering applied to the damaged tissue showed three clusters, two of which were similar to the clusters found in the healthy tissue. For the additional cluster, spectral characteristics of both collagen and muscle were found, indicating the presence of collagen infiltration surrounding muscle fibers which was not present in normal tissue. Collagen infiltration is often associated with structural damage in the bladder, however to determine the extent of the collagen infiltration it was necessary to remove the collagen that naturally surrounds muscle fibers. Raman spectra also showed an increase in lactate $(857,1415,1455$ $\left.\mathrm{cm}^{-1}\right)$ and glycogen $\left(857,1005,1079,1321,1447,1659 \mathrm{~cm}^{-1}\right)$ in the damaged tissue which the authors suggest might indicate that ischemia is related to increased glycogen levels and collagen infiltration. In this study, de Jong et al. [52] again demonstrated the use of Raman spectroscopic mapping for investigating the biochemistry of bladder wall tissue, and they indicated how its ability to detect changes in molecular composition could be utilized as a diagnostic tool.

Prieto et al. [53] extended on from the work of de Jong et al. [52] in the analysis of bladder wall layers using Raman spectroscopy, but instead of using the Raman mapping technique, Prieto used a Kerr-gated Raman spectroscopy procedure consisting of a pulsed laser, two crossed polarizers and a 4 ps optical Kerr shutter. A normal human bladder sample, obtained via biopsy during a cystoscopic procedure, snap-frozen and stored at $-80^{\circ} \mathrm{C}$ prior 

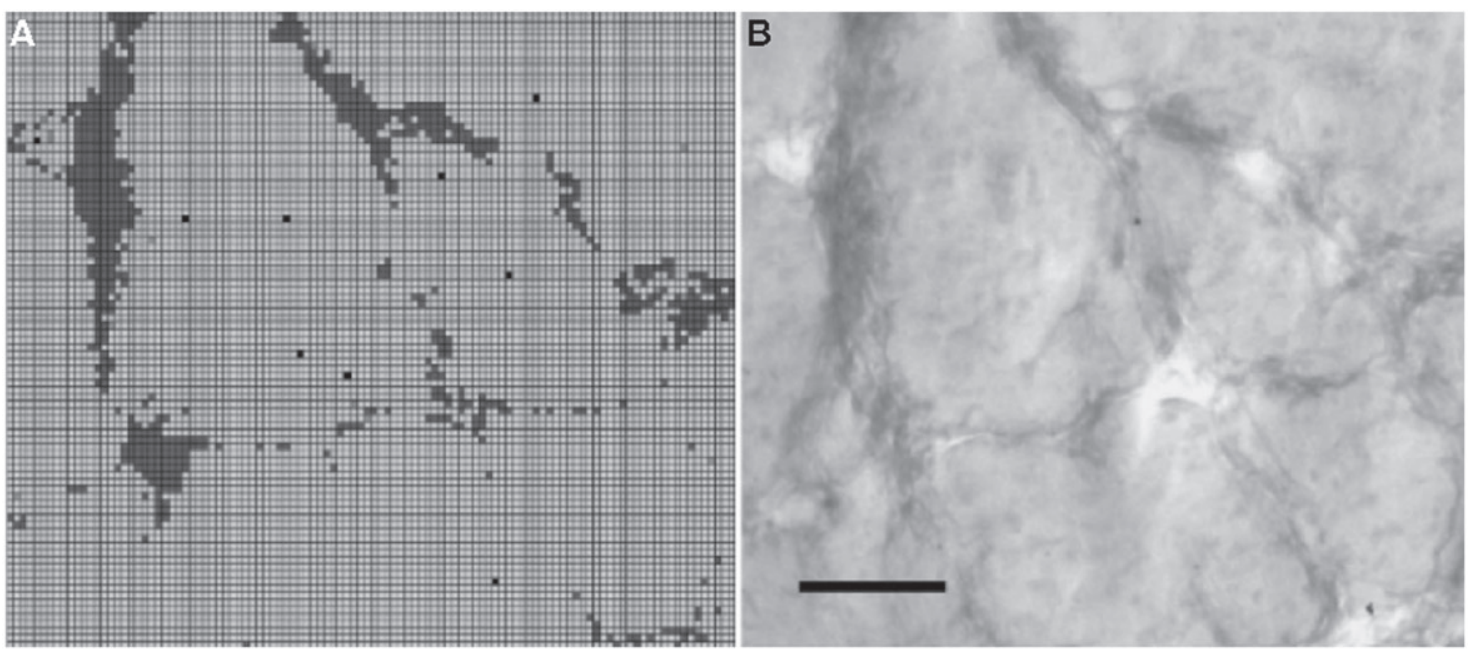

Figure 10 Raman map (A) and digital image stained with H\&E (B) of a section of normal bladder tissue showing similarities between tissue regions. Images obtained from de Jong et al. [52], reproduced with permission from Elsevier.

to measurements, was analyzed using this Kerr-gated set-up on a quartz cell substrate containing uric acid. This technique allows for spectra to be obtained from different depths in the tissue as signals emerge from the tissue at different times depending on their penetration depth. Therefore, changes in shift peak intensities were observed as the temporal position of the Kerr gate was varied which could then be related to different depth measurements with acquisition times, which correspond to the urothelium, lamina propria and muscle layer, and eventually, all tissue signals were lost and uric acid peaks appeared (662 and $803 \mathrm{~cm}^{-1}$ ). Whilst this depth profiling method showed potential for the investigation of bladder wall layers and ability to assess bladder pathology, it was reported that the instrumentation (which filled two rooms) was too large to be used in a hospital setting in the near future.

In 2006, de Jong et al. [45] used a combination of Raman spectroscopic mapping and compositional analysis to evaluate the main molecular differences between tumor and non-tumor bladder tissue and gain insight into the biochemical alterations that are associated with pathogenesis. Raman spectroscopic maps were generated from 15 bladder tissue specimen, five of which were nontumor (normal and cystitis) and the remaining 10 were tumor tissues (TIS, T1 and T2), with a signal collection time of $20 \mathrm{~s}$. To perform compositional analysis on these spectra, a large reference database of component spectra was employed, Raman spectra of 18 different compounds were used in total; collagen I, collagen III, actin, albumin, DNA, ribonucleic acid (RNA), cholesterol, $\beta$-carotene, gangliosides, glycogen, phosphatidylcholine, phosphatidylethanolamine, arachidonic acid, palmitic acid, palmitoleic acid, stearic acid, oleic acid methyl ester and linoleic acid. Wavenumber calibration and baseline correction were used for pre-processing the Raman spectra before being clustered into CAS, representing tumor and non-tumor groups. Each CAS was input to the compositional analysis using the reference database, and it was found that (i) collagen I, collagen III and some lipids are more expressed in non-tumor clusters, (ii) $\beta$-carotene was not fitted significantly differently in tumor and non-tumor clusters, and (iii) the rest of the components are more expressed in the tumor clusters. RNA and DNA contents were found to be higher in tumor regions compared to non-tumor tissue. Inflammation areas (non-tumor) were also found to express abundant levels of RNA and DNA. To the best of our knowledge, this study represents the first Ramanbased compositional analysis of the main biomolecular differences between tumor and non-tumor bladder tissue.

In 2007, Stone and colleagues [54] continued on from this work to determine the biochemical differences associated with different bladder pathological groups. Conventional Raman spectra were obtained from 73 patient samples in vitro consisting of five different bladder pathology groups; normal, cystitis, CIS, G1/G2 TCC and G3 TCC. All samples were obtained during TURP and TURBT, snap-frozen and stored at $-80^{\circ} \mathrm{C}$, before returning to room temperature for measurement. These spectra were subjected to compositional analysis using a reference database similar to that used by de Jong et al. [45]. To generate the reference database, Raman spectra were obtained from the following constituents; actin, $\beta$-carotene, collagen I, collagen III, triolein, choline, oleic acid, DNA and cholesterol. Raman spectra from the different bladder 


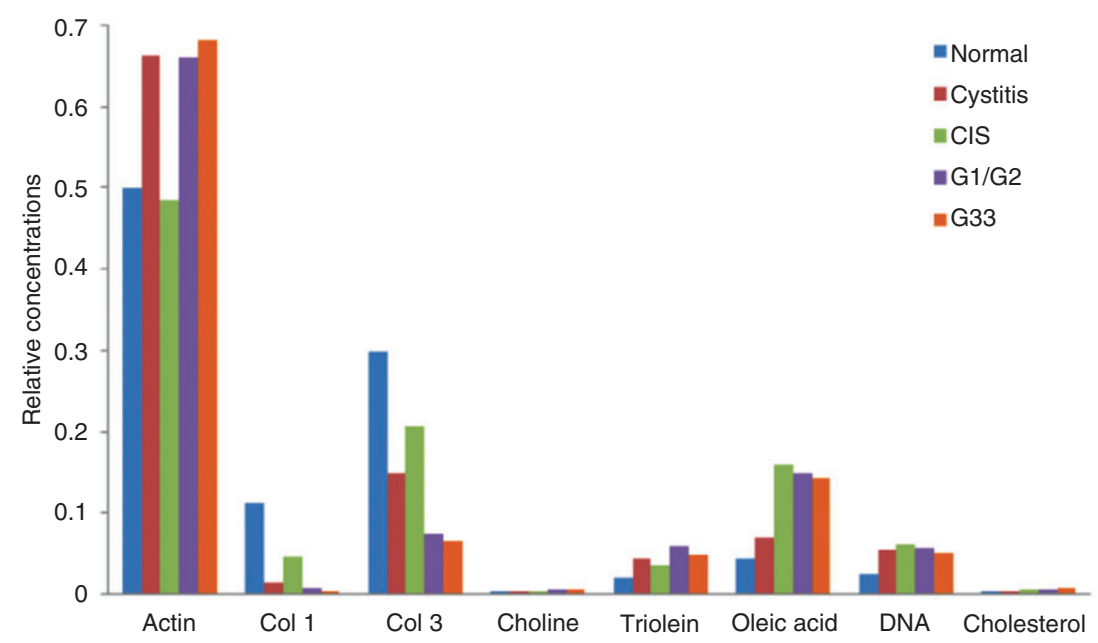

Figure 11 Bar graph representing the data presented by Stone et al. [54] showing the relative concentrations of each constituent associated with each bladder pathology.

pathologies were initially pre-processed, and then fitted to the reference database using OLS. Results of this study can be seen in Figure 11, which shows an increase in DNA content as the bladder tissue progresses from normal to malignant. An increase is also seen with cholesterol, choline, oleic acid and triolein with tissue progression. Actin levels vary throughout the pathological groups, and there is a decrease in both collagen I and collagen III with disease progression. This mirrors the biochemical information gathered by de Jong et al. [45] and similarly enables a better understanding of the progress of bladder disease which could potentially be used to eliminate intraand inter-observer error in histopathology.

In 2009, Grimbergen et al. [11] investigated the effects of the photosensitive drug 5-ALA on the biochemical analysis of bladder tissue using conventional Raman spectroscopy. The motivation for this study was the potential improvement in the diagnostic capability of bladder pathology by combining Raman spectroscopy and fluorescent imaging, the latter enabling a more targeted application of the former. Raman spectra were obtained from benign and malignant bladder samples both with and without the presence of 5-ALA (92 biopsies from 73 patients without 5-ALA and 38 biopsies from 19 patients with 5-ALA), whereby benign samples represented normal and cystitis, and malignant samples included CIS, G1 TCC, G2 TCC and G3 TCC. From the recorded spectra, it was noted that the key spectral regions for discriminating between the biochemistry of benign and malignant bladder tissue samples in the presence of 5-ALA were found over the following ranges: 422-466, 530-574, 634-698, 10301070, 1170-1200, 1250-1290, 1400-1480 and 1550-1610 $\mathrm{cm}^{-1}$. However, since the goal of 5-ALA is to increase the concentration of the photosensitizer PpIX in the cancerous tumor regions, it is possible that this photosensitizer is affecting the biochemistry of the tissue, resulting in alterations to the Raman spectra obtained with 5-ALA in comparison to those without any photosensitizer. So whilst this study has shown the potential application of Raman spectroscopy with fluorescence for discriminating between the biochemistry of benign and malignant bladder tissue, it should be noted that the differences that were observed do not reflect the same molecular differences that are seen in the biochemistry of bladder samples without 5-ALA. Despite this, there is still significant evidence to show that the combination of Raman spectroscopy with fluorescence-guided cystoscopy could be used to improve the diagnosis of bladder cancer.

In 2010, Draga and colleagues [8] investigated the feasibility of using a Raman spectroscopic probe to detect differences in the biochemistry of benign and malignant bladder tissue in vivo with a high-volume fiber-optic probe, and also to be able to determine the invasiveness of the disease. Raman spectra were obtained from normal and cancerous (graded Ta, T1 and T2 by experienced pathologists) bladder lesions, from 38 patients, in vivo during TURBT procedures with integration times between 1-5 s. From these spectra, the most prominent peaks found in normal bladder tissue were at $875,1080,1289,1303,1446$ and $1657 \mathrm{~cm}^{-1}$ which relate to lipids and proteins, with the peak at $875 \mathrm{~cm}^{-1}$ representing hydroxyproline, an important component of collagen. Peaks found in the bladder cancer spectra were at $680,789,1003,1170,1180,1208,1370,1386,1560$ and $1610 \mathrm{~cm}^{-1}$, mainly associated with amino acids (such as phenylalanine and tryptophan) and DNA. This agrees 
with previous studies where a decrease in collagen and an increase in DNA and lipids is observed as tissue progresses from normal to cancerous. Inflammation was distinguished from normal and cancer tissue in the following wavenumber peaks: 562, 572, 1485 and $1569 \mathrm{~cm}^{-1}$.

A further breakdown of the spectral peaks observed can be seen in Table 15. The high-volume Raman probe used in this study measured up to a depth of $2 \mathrm{~mm}$ which means that most of the signal collected was from the submucosa and muscle layer, with significantly less contribution coming from the submucosal fatty layers. It was proposed, therefore, that the spectral features of lipids observed could potentially be used to assess the invasiveness of the tumor. This study achieved, for the first time, the goal of investigating the biochemistry of different bladder tissue pathologies in vivo using a real-time, nondestructive method in a clinical setting and will undoubtedly pave the way for the future development of novel fiber-optic Raman probes that will aid in the understanding of the etiology of bladder cancer.

Most recently, in 2011, as part of a proof-of-concept study using bladder tissue, Shapiro et al. [12] applied Raman spectroscopy to deparaffinized bladder samples (three normal samples, six with Ta, four with T2 and two samples with TIS; obtained from cystectomy and prostatectomy procedures) to acquire Raman spectra for each pathology. From these spectra, a distinct peak was found at $1584 \mathrm{~cm}^{-1}$ that is only present in bladder carcinoma tissue, which had not been previously reported. Raman spectra of both low-grade and high-grade cancer show this peak at $1584 \mathrm{~cm}^{-1}$ which was not seen in spectra from benign tissue. It was also reported that the height of this peak correlated with the carcinoma grade of the tissue, however the peak was not assigned to any particular biomolecular components. This distinctive peak seems to not only be an excellent method for the diagnosis of bladder cancer, but also for determining the associated pathological grade.

\section{Conclusion and future developments}

It is clear from the results reviewed in this paper that there is growing potential for the application of Raman spectroscopy to the study of urinary bladder disease in order to improve patient treatment and patient outcome. The technique can be applied in a number of different forms, including conventional Raman microspectroscopy, as well as using fiber-optic probes. Raman microspectroscopy has been shown to be a strong candidate for improving the diagnostic sensitivity and specificity of bladder cytology, with particular interest for the case of low-grade cancer cells, for which a recent (and the first) patient study showed a sensitivity and specificity of $92 \%$ and 91\% respectively. Raman fibers have been shown to return similar results for both in vitro/ex vivo and in vivo samples. The improvement in sensitivity over existing diagnostic procedures is not only due to the additional biochemical information provided by the Raman spectrum and the ability to classify this using multivariate diagnostic algorithms, but it is also partially due to the reduction in interand intra-observer variation, which can vary by up to $45-50 \%$ using current clinical methods [20,56]. As well as improved diagnostic accuracy, Raman spectroscopy also enables an estimation of the biomolecular composition of tissue and cell samples, therefore facilitating further understanding of the etiology of bladder disease and the biochemical continuum of disease progression.

Despite the various positive results presented in this paper, it should be noted that the use of Raman spectroscopy for bladder diagnostics is not without its problems. While the method has been demonstrated to produce accurate results in real time, further development is necessary to ensure that these systems are sufficiently robust for everyday medical usage. Presently, the application of Raman microspectroscopy to cells and tissue requires an experienced hand and there are numerous practical issues that need to be addressed, for example the 3-D point in a cell from which the spectrum is acquired is important, as is the need to periodically calibrate the system for wavelength shifts.

Acquisition time is usually in the order of $30 \mathrm{~s}$ which can be an issue if many cells or tissue locations are to be examined, and different substrates (or fibers) can provide different background signals that can provide sharply varying results. Praveen et al. [49] recently applied modulated Raman spectroscopy, a method used to remove unwanted background signals from Raman spectra, with a significantly reduced acquisition time ( $6 \mathrm{~s}$ per measurement), and was able to completely discriminate between healthy and cancerous cells. Whilst this study has shown that it may be possible to develop high throughput cell screening methods with this technique, there are other experimental parameters which need to be considered to fully optimize the application of Raman spectroscopy for cancer diagnostics.

The lack of standardization, in terms of equipment, consumables and methodologies has resulted in the recording of significantly differing spectra in the studies to date and this has prevented the use of training datasets 
being shared across research groups and has inhibited the comparison of results in a controlled manner. Even identical systems and methodologies can produce moderately varying spectra for the same sample, which may be attributed to the relatively strong noise signal and variation in experimental settings on a precise scale. If Raman spectroscopy is ever to become an everyday clinical tool it is essential that a standardized procedure will be established in order to overcome these inconsistencies. One solution may be an automation of the process insofar as possible in order to reduce user variability.

While it is essential for further research and development to focus on minimizing problems relating to consistency, it is expected that the areas that will receive the greatest attention going forward will be Raman cystoscopy and Raman cytology. Raman cytoscopy probes not only have the potential to detect and classify the presence of disease, but they may also be applied during surgical procedures to "map" regions of disease versus healthy tissue as well as profiling the depth of the tumor. It is most likely that numerous research groups will attempt to design Raman fiber-optic probes that overcome the problems of being too large and providing a too weak signal.

One of the greatest problems that needs to be overcome is the strong background signal from the fiber itself. A strong candidate for solving these problems is photonic crystal fibers. These recently developed fibers could be used to overcome the technical challenge of packing multiple fibers into a small probe and because they employ a hollow core, and in addition these fibers do not add any contribution to the Raman signal like those seen with silica fibers [29]. Another possible solution is the combination of modulated Raman spectroscopy with a fiber-optic probe which has been shown to suppress the fluorescent background signals generated within the fiber, thus allowing for greater fidelity of the Raman signal [28]. Many more in vivo Raman cystoscopy experiments are expected in the near future.

Similarly, further progress could be made to improve Raman cytology. Following from the first patient study based on cells obtained from voided urine in 2011 [12], which demonstrated promising results, it is expected that similar studies will try to build on this. One avenue of research will be the application of modulated Raman spectroscopy in a patient study to improve on the diagnostic accuracy achieved with conventional Raman microspectroscopy. Another possibility is the application of surface-enhanced Raman spectroscopy (SERS) to bladder cells. SERS is a technique used to amplify the Raman signal from cells (or tissue), as explained in more detail in [57], which has the potential to be integrated into a point-of-care device for rapid cell analysis and diagnosis. A simple approach is to mix a solution of cells with a solution of gold nanoparticles, and in regions of the cell where aggregations of nanoparticles occur, it is possible to obtain a significantly (approximately million fold) enhanced Raman signal. Though SERS is known to have problems regarding reproducibility, it has been recently applied to urine samples for point-of-care diagnosis of urinary tract infections [58], and may have the potential to be further developed to detect other urinary diseases. One of the great advantages that such an approach may have would be the relatively strong power of the Raman signal which would therefore allow for a much shorter acquisition time. This feature may facilitate the use of microfluidic channels to detect the spectra from fast moving cells in a urine sample. Another possibility for future research is the automation of Raman spectroscopy for cytology, which involves specialist digital image processing techniques to detect the nucleus within "suspicious" cells, obtaining Raman spectra from these targeted nuclei, and using diagnostic algorithms to classify the pathological state of the cells being measured. Recently the first such automated system has been developed [59] in the form of a user friendly graphical user interface which has been applied successfully for cervical cancer screening. It may be possible to integrate a similar system into bladder cancer diagnostics to automate (and standardize) the Raman spectroscopic method.

Acknowledgments: This research was conducted with the financial support of Science Foundation Ireland (SFI) under Grant Number 11/SIRG/I2140.

Received February 19, 2014; revised April 11, 2014; accepted April 15, 2014

[3] Khochikar MV. Rationale for an early detection program for bladder cancer. Indian J Urol 2011;27(2):218-25.

[4] Wallace DM, Bryan RT, Dunn JA, Begum G, Bathers S; West Midlands Urological Research Group. Delay and survival in bladder cancer. Br J Urol Int 2002;89(9):868-78. 
[5] Hanchanale VS, Rao AR, Das S. Raman spectroscopy and its urological applications. Indian J Urol 2008;24(4):444-50.

[6] Kallaway C, Almond LM, Barr H, Wood J, Hutchings J, Kendall C, Stone N. Advances in the clinical application of Raman spectroscopy for cancer diagnostics. Photodiagnosis Photodyn Ther 2013;10(3):207-19.

[7] Cauberg Evelyne CC, de la Rosette JJ, de Reijke TM. Emerging optical techniques in advanced cystoscopy for bladder cancer diagnosis: A review of the current literature. Indian J Urol 2011;27(2):245-51.

[8] Draga RO, Grimbergen MC, Vijverberg PL, van Swol CF, Jonges TG, Kummer JA, Ruud Bosch JL. In vivo bladder cancer diagnosis by high-volume Raman spectroscopy. Anal Chem 2010;82(14):5993-9.

[9] Barman I, Dingari NC, Singh GP, Kumar R, Lang S, Nabi G. Selective sampling using confocal Raman spectroscopy. provides enhanced specificity for urinary bladder cancer diagnosis. Anal Bioanal Chem 2012;404(10):3091-9.

[10] Witjes JA, Douglass J. The role of hexaminolevulinate fluorescence cystoscopy in bladder cancer. Nat Clin Pract Urol 2007;4(10):542-9.

[11] Grimbergen MC, van Swol CF, van Moorselaar RJ, Uff J, Mahadevan-Jansen A, Stone N. Raman spectroscopy of bladder tissue in the presence of 5-aminolevulinic acid. J Photochem Photobiol B 2009;95(3):170-6.

[12] Shapiro A, Gofrit ON, Pizov G, Cohen JK, Maier J. Raman.. molecular imaging: a novel spectroscopic technique for diagnosis of bladder cancer in urine specimens. Eur Urol 2011;59(1):106-12.

[13] Canetta E, Mazilu M, De Luca AC, Carruthers AE, Dholakia K, Neilson S, Sargeant H, Briscoe T, Herrington CS, Riches AC. Modulated Raman spectroscopy for enhanced identification of bladder tumor cells in urine samples. J Biomed 0 pt 2011;16(3):037002.

[14] Shariat SF, Karam JA, Lotan Y, Karakiewizc PI. Critical evaluation of urinary markers for bladder cancer detection and monitoring. Rev Urol 2008;10(2):120-35.

[15] Mowatt G, N'Dow J, Vale L, Nabi G, Boachie C, Cook JA, Fraser C, Griffiths TR; Aberdeen Technology Assessment Review (TAR) Group. Photodynamic diagnosis of bladder cancer compared with white light cystoscopy: Systematic review and meta-analysis. Int J Technol Assess Health Care 2011;27(1):3-10.

[16] Walsh PC, Retik AB, Vaughan Jr ED, Wein AJ, Kavoussi LR, Novick AC, Partin AW, Peters CA, editors. Campbell's Urology. 8th edition. Volume 4. Philadelphia: Elsevier Saunders; 2002.

[17] Siegel R, Ward E, Brawley O, Jemal A. Cancer statistics, 2011: the impact of eliminating socioeconomic and racial disparities on premature cancer deaths. CA Cancer J Clin 2011;61(4): 212-36.

[18] Heney NM. Natural history of superficial bladder cancer. Prognostic features and long-term disease course. Urol Clin North Am 1992;19(3):429-33.

[19] MacVicar D, editor. Carcinoma of the bladder. Cambridge: Cambridge University Press; 2008.

[20] Crow P, Stone N, Kendall CA, Persad RA, Wright MP. Optical diagnostics in urology: current applications and future prospects. Br JUrol Int 2003;92(4):400-7.

[21] Babjuk M, Burger M, Zigeuner R, Shariat SF, van Rhijn BW, Compérat E, Sylvester RJ, Kaasinen E, Böhle A, Palou
Redorta J, Rouprêt M. EAU guidelines on non-muscle-invasive urothelial carcinoma of the bladder: update 2013. Eur Urol 2013;64(4):639-53.

[22] Saleh BEA, Teich MC. Fundamentals of photonics. 2nd ed. Hoboken: John Wiley \& Sons, Inc.; 2007.

[23] Mazilu M, De Luca AC, Riches A, Herrington CS, Dholakia K. Optimal algorithm for fluorescence suppression of modulated. Raman spectroscopy. Opt Express 2010;18(11):11382-95.

[24] Utzinger U, Richards-Kortum RR. Fiber optic probes for biomedical optical spectroscopy. J Biomed Opt 2003;8(1):121-47.

[25] de Lima CJ, Sathaiah S, Silveira L, Zângaro RA, Pacheco MT. Development of catheters with low fiber background signals for Raman spectroscopic diagnosis applications. Artif Organs 2000;24(3):231-4.

[26] Day JC, Stone N. A subcutaneous Raman needle probe. Appl Spectrosc 2013;67(3):349-54.

[27] Koljenović S, Bakker Schut TC, Wolthuis R, de Jong B, Santos L, Caspers PJ, Kros JM, Puppels GJ. Tissue characterization using high wave number Raman spectroscopy. J Biomed Opt 2005;10(3):031116.

[28] Praveen BB, Ashok PC, Mazilu M, Riches A, Herrington S, Dholakia K. Fluorescence suppression using wavelength modulated Raman spectroscopy in fiber-probe-based tissue analysis. J Biomed Opt 2012;17(7):077006.

[29] Ghenuche P, Rammler S, Joly NY, Scharrer M, Frosz M, Wenger J, Russell PS, Rigneault H. Kagome hollow-core photonic crystal fiber probe for Raman spectroscopy. Opt Lett 2012;37(21):4371-3.

[30] Ashkin A. Acceleration and trapping of particles by radiation pressure. Phys Rev Lett 1970;24:156-9.

[31] Neuman KC, Block SM. Optical trapping. Rev Sci Instrum 2004;75(9):2787-809.

[32] Harvey TJ, Hughes C, Ward AD, Faria EC, Henderson A, Clarke NW, Brown MD, Snook RD, Gardner P. Classification of fixed. urological cells using Raman tweezers. J Biophotonics 2009;2(1-2):47-69.

[33] Fore S, Chan J, Taylor D, Huser T. Raman spectroscopy of. individual monocytes reveals that single-beam optical trapping of mononuclear cells occurs by their nucleus. J Opt 2011;13(4):44021.

[34] Bankapur A, Zachariah E, Chidangil S, Valiathan M, Mathur D. Raman tweezers spectroscopy of live, single red and white blood cells. PLoS One 2010;5(4):e10427.

[35] Harvey TJ, Faria EC, Henderson A, Gazi E, Ward AD, Clarke NW, Brown MD, Snook RD, Gardner P. Spectral discrimination of. live prostate and bladder cancer cell lines using Raman optical. tweezers. J Biomed Opt 2008;13(6):064004.

[36] Harvey TJ, Hughes C, Ward AD, Gazi E, Correia Faria E, Clarke NW, Brown M, Snook R, Gardner P. The use of Raman tweezers and chemometric analysis to discriminate the urological cell lines, PC-3, LNCaP, BPH and MGH-U1. In: Vaidyan VK, Jayakumar VS, editors. Perspectives in vibrational spectroscopy. Proceedings of the 2nd International Conference on Perspectives in Vibrational Spectroscopy (ICOPVS 2008). AIP Conference Proceedings Volume 1075. Melville: American Institute of Physics Publishing; 2008, p. 138-40.

[37] Harvey T, Hughes C, Ward AD, Gazi E, Correia Faria E, Snook R, Brown M, Clarke NW, Gardner P. Analysis of prostate and bladder cells using Raman tweezers. In: Withnall B, Chowdhry BZ. Proceedings of the XX1st International Conference on 
Raman Spectroscopy. Chichester: IMP Publications LLP; 2008, p. 1015-17.

[38] Wolthuis R, Bakker Schut TC, Caspers PJ, Buschman HPJ, Römer TJ, Bruining HA, Puppels GJ. Raman spectroscopic methods for in vitro and in vivo tissue characterization. In: Mason WT, editor. Fluorescent and luminescent probes for biological activity. A practical guide to technology for quantitative real-time analysis. Second edition. London and San Diego: Academic Press; 1999, p. 433-55.

[39] Liland KH, Almøy T, Mevik BH. Optimal choice of baseline correction for multivariate calibration of spectra. Appl Spectrosc 2010;64(9):1007-16.

[40] Afseth NK, Segtnan VH, Wold JP. Raman spectra of biological samples: A study of pre-processing methods. Appl Spectrosc 2006;60(12):1358-67.

[41] Huang J, Romero-Torres S, Moshgbar M. Practical considerations in data pre-treatment for NIR and Raman spectroscopy. http://www.americanpharmaceuticalreview.com/FeaturedArticles/116330-Practical-Considerations-in-Data-Pre-treatment-for-NIR-and-Raman-Spectroscopy/ [Accessed on March 27, 2014].

[42] De Luca AC, Mazilu M, Riches A, Herrington CS, Dholakia K. Online fluorescence suppression in modulated Raman spectroscopy. Anal Chem 2010;82(2):738-45.

[43] Stone N, Kendall C, Shepherd N, Crow P, Barr H. Near-infrared Raman spectroscopy for the classification of epithelial precancers and cancers. J Raman Spectrosc 2002;33(7):564-73.

[44] Crow P, Uff JS, Farmer JA, Wright MP, Stone N. The use of Raman spectroscopy to identify and characterize transitional cell carcinoma in vitro. Br J Urol Int 2004;93(9):1232-6.

[45] de Jong BW, Schut TC, Maquelin K, van der Kwast T, Bangma $\mathrm{CH}$, Kok DJ, Puppels GJ. Discrimination between non-tumor bladder tissue and tumor by Raman spectroscopy. Anal Chem 2006;78(22):7761-9.

[46] Crow P, Molckovsky A, Stone N, Uff J, Wilson B, WongKeeSong LM. Assessment of fiberoptic near-infrared raman spectroscopy for diagnosis of bladder and prostate cancer. Urology 2005;65(6):1126-30.

[47] Grimbergen MCM, van Swol CFP, Draga ROP, van Diest P, Verdaasdonk RM, Stone N, Bosch JHLR. Bladder cancer diagnostics during cystoscopy using Raman spectroscopy. Proc SPIE 2009;7161:716114.

[48] O'Toole CM, Povey S, Hepburn P, Franks LM. Identity of some human bladder cancer cell lines. Nature 1983;301(5899):429-30.
[49] Praveen BB, Mazilu M, Marchington RF, Herrington CS, Riches A, Dholakia K. Optimisation of wavelength modulated Raman spectroscopy: towards high throughput cell screening. PLoS One 2013;8(6):e67211.

[50] Feld MS, Manoharan R, Salenius J, Orenstein-Carndona J, Roemer TJ, Brennan III JF, Dasari RR, Wang Y. Detection and characterization of human tissue lesions with near-infrared Raman spectroscopy. Proc SPIE 1995;2388:99-104.

[51] de Jong BW, Bakker Schut TC, Wolffenbuttel KP, Nijman JM, Kok DJ, Puppels GJ. Identification of bladder wall layers by Raman spectroscopy. J Urol 2002;168(4 Pt 2):1771-8.

[52] de Jong BWD, Bakker Schut TC, Coppens J, Wolfenenbuttel KP, Kok DJ, Puppels GJ. Raman spectroscopic detection of changes in molecular composition of bladder muscle tissue caused by outlet obstruction. Vib Spectrosc 2003;32(1):57-65.

[53] Prieto MC, Matousek P, Towrie M, Parker AW, Wright M, Ritchie AW, Stone N. Use of picosecond Kerr-gated Raman spectroscopy to suppress signals from both surface and deep layers in bladder and prostate tissue. J Biomed 0pt 2005;10(4):44006.

[54] Stone N, Hart Prieto MC, Crow P, Uff J, Ritchie AW. The use of Raman spectroscopy to provide an estimation of the gross biochemistry associated with urological pathologies. Anal Bioanal Chem 2007;387(5):1657-68.

[55] Movasaghi Z, Rehman S, Rehman IU. Raman spectroscopy of biological tissues. Appl Spectrosc Rev 2007;42(5):493-541.

[56] Tosoni I, Wagner U, Sauter G, Egloff M, Knönagel H, Alund G, Bannwart F, Mihatsch MJ, Gasser TC, Maurer R. Clinical significance of interobserver differences in the staging and grading of superficial bladder cancer. $\mathrm{Br} J$ Urol Int 2000;85(1):48-53.

[57] Palonpon AF1, Ando J, Yamakoshi H, Dodo K, Sodeoka M, Kawata S, Fujita K. Raman and SERS microscopy for molecular imaging of live cells. Nat Protoc 2013;8(4):677-92.

[58] Hadjigeorgiou K, Kastanos E, Kyriakides A, Pitris C. Point-ofcare diagnosis of urinary tract infection (UTI) using surface enhanced Raman spectroscopy (SERS). Proceedings of the 2012 IEEE 12th International Conference on Bioinformatics and Bioengineering; 2012, p. 333-7.

[59] Blackledge J, Dubovitskiy D, Lyng F. Targeting cell nuclei for the automation of Raman spectroscopy in cytology. ISAST Transactions on Computers and Intelligent Systems 2012;4(1):42-51. http://users.jyu.fi/ timoh/isast2012.pdf [Accessed on March 27, 2014]. 